Space weathering simulations through controlled growth of iron nanoparticles on olivine

Kohout, T.

2014

pÿKohout , T, uda , J , Filip , J , Britt , D , Bradley , T, Tuek , J , Skála , R, Kletetschka , G , pÿKaalík , J , Malina , O , 'iaková , K \& ZboYil , R 2014 , ' Space weathering simulations through controlled growth of iron nanoparticles on olivine ' , Icarus , vol. 273 , pp. 75-83 . https://doi.org/10.1016/j.ica

http://hdl.handle.net/10138/136213

https://doi.org/10.1016/j.icarus.2014.04.004

acceptedVersion

Downloaded from Helda, University of Helsinki institutional repository.

This is an electronic reprint of the original article.

This reprint may differ from the original in pagination and typographic detail.

Please cite the original version. 


\title{
Space weathering simulations through controlled growth of iron nanoparticles on
}

\section{olivine}

T. Kohout ${ }^{1,2}$ (kohout@gli.cas.cz), J. Čuda ${ }^{3}$, J. Filip ${ }^{3}$, D. Britt ${ }^{4}$, T. Bradley ${ }^{4}$, J. Tuček ${ }^{3}$, R. Skála ${ }^{1}$, G. Kletetschka ${ }^{1,5}$, J. Kašlík ${ }^{3}$, O. Malina ${ }^{3}$, K. Šišková ${ }^{3}$, and R. Zbořill

1. Institute of Geology, Academy of Sciences of the Czech Republic, Prague, Czech Republic

2. Department of Physics, University of Helsinki, Finland

3. Regional Centre of Advanced Technologies and Materials, Faculty of Science, Departments of Experimental Physics and Physical Chemistry, Palacký University, Olomouc, Czech Republic

4. Department of Physics, University of Central Florida, Orlando, USA

5. Faculty of Science, Charles University in Prague, Czech Republic

\begin{abstract}
Airless planetary bodies are directly exposed to space weathering. The main spectral effects of space weathering are darkening, reduction in intensity of silicate mineral absorption bands, and an increase in the spectral slope towards longer wavelengths (reddening). Production of nanophase metallic iron $\left(\mathrm{npFe}^{0}\right)$ during space weathering plays major role in these spectral changes. A laboratory procedure for the controlled production of $\mathrm{npFe}^{0}$ in silicate mineral powders has been developed. The method is based on a two-step thermal treatment of low-iron olivine, first in ambient air and then in hydrogen atmosphere. Through this process, a series of olivine powder samples was prepared with varying amounts of $n \mathrm{nFe}^{0}$ in the $7-20 \mathrm{~nm}$ size range. A logarithmic trend is observed between amount of $n \mathrm{nFe}^{0}$ and darkening, reduction of $1 \mu \mathrm{m}$ olivine absorption band, reddening, and $1 \mu \mathrm{m}$ band width. Olivine with a population of physically larger $n p F e^{0}$
\end{abstract}


particles follows spectral trends similar to other samples, except for the reddening trend. This is interpreted as the larger, $\sim 40-50 \mathrm{~nm}$ sized, $\mathrm{npFe}^{0}$ particles do not contribute to the spectral slope change as efficiently as the smaller $n p F e^{0}$ fraction. A linear trend is observed between the amount of $\mathrm{npFe}^{0}$ and $1 \mu \mathrm{m}$ band center position, most likely caused by $\mathrm{Fe}^{2+}$ disassociation from olivine structure into $\mathrm{npFe}^{0}$ particles.

\section{Introduction}

Airless planetary bodies are directly exposed to the space environment and thus to space weathering. Space weathering is caused by a combination of solar wind and solar radiation, micrometeorite bombardment, and cosmic radiation that can alter the physical, chemical, and crystallographic properties of airless regoliths. Among other effects, space weathering causes changes in the visible, IR and UV spectra of exposed surface material making it difficult to compare weathered asteroidal and lunar spectra to those of unweathered silicate minerals, meteorites, and lunar samples.

\section{Lunar type space weathering}

Lunar samples delivered to Earth by the Apollo program during the 1960's and 1970's significantly contributed to our understanding of space weathering. It was the first time that direct laboratory studies were possible on a material exposed for long periods to the space environment and, therefore, modified by space weathering. Numerous early studies (e.g. Adams and Jones, 1970, McCord and Johnson, 1970, Adams and McCord, 1971, 1973, McCord and Adams, 1973) found that, compared to artificially crushed fresh Apollo rock samples, spectra of lunar soils are darker, have reduced intensity of silicate mineral absorption bands, and their spectral slope increases towards longer wavelengths (reddening). 
Reviews by Hapke (2001) or Chapman (2004) provide an understanding of the mechanisms involved in space weathering. Earlier lunar soil studies indicated that space weathering may cause an accumulation of dark agglutinitic glass formed by surface melting of regolith by micrometeorite impacts (Adams and McCord, 1971). Agglutinitic glass is an important optical agent influencing reflectance spectra of lunar regolith. However, its presence could not explain all of the spectral discrepancies in lunar soils and planetary surfaces, especially the spectral reddening (Pieters et al., 1993). Hapke et al. (1975) suggested that optical effects of space weathering may be caused by metallic submicroscopic iron particles (SM Fe), also referred to as nanophase iron (npFe ${ }^{0}$ ) particles, within vapor deposition rims on lunar soil grains. This $\mathrm{npFe}^{0}$ is believed to originate from disassociation of Fe from Fe-bearing minerals through solar wind sputtering and micro-impactgenerated vapor (e.g. Keller and McKay, 1993; 1997, Hapke, 2001), and its concentration is highest in the fine fraction of lunar soils (Pieters et al, 1993; Taylor et al., 2001). Support for this theory came later from transmission electron microscopy (TEM). TEM observations showed the presence of such npFe ${ }^{0}$ particles in lunar soil grain coatings (e.g. Keller and M cKay, 1997; Hapke, 2001). The typical size range of $\mathrm{npFe}^{0}$ particles is $\sim 1-10 \mathrm{~nm}$ in vapor deposition rims and $\sim 10-100 \mathrm{~nm}$ in agglutinates (e.g. Keller and Clemett, 2001).

\section{S-type asteroid space weathering}

As in the case of lunar rocks, spectral discrepancies are also observed between meteorites and asteroids (e.g. Hapke, 2001 and Chapman, 2004). Silicate rich S-complex asteroids are represented by absorption bands characteristic of olivine (at wavelengths around $1 \mu \mathrm{m}$ ) and pyroxene (at wavelengths around 1 and $2 \mu \mathrm{m}$ ). Spectra of ordinary chondrite meteorites contain similar absorption bands, but, compared to asteroids, the bands in meteorites are usually more intense and the overall spectral slope is flatter. Numerous laboratory simulations (involving laser 
irradiation and ion bombardment, e.g. Brunetto et al., 2005) of ordinary chondrites and detailed spectral observations of asteroids and meteorites (e.g. Marchi et al., 2005; Lazzarin et al., 2006) show that space weathering effects on chondritic materials and S-complex asteroids display similarities to lunar soils where solar wind ion bombardment induces atom displacements and micrometeorite bombardment forms $\mathrm{npFe}^{0}$ particles. Direct evidence of $\mathrm{npFe}{ }^{0}$ particle formation on the surface of S-type asteroids was recently reported by Noguchi et al. (2011) in a study of surface coatings of regolith grains obtained from asteroid 25143 Itokawa by the Hayabusa sample return mission. However, compared to lunar weathering, the mechanism of asteroidal space weathering is more complex and its various effects (e.g. absorption band weakening, darkening, and reddening) do occur in spectra, with a highly varying intensity, even on the same asteroids (e.g. Chapman, 2004; Hiroi et al., 2006; Gaffey, 2010).

Observations of young asteroid families shows that space weathering occurs relatively rapidly, within $10^{6}$ years after the breakup of the parent body (Vernazza et al., 2009). Similar to lunar type weathering, space weathering on asteroids affects a thin surface regolith layer only and this layer can be disturbed by geological processes such as landslides, as observed on Eros by Clark et al. (2001), or by tidal forces during close planetary encounters (Binzel et al., 2010).

Space weathering laboratory simulations

Laboratory production of npFe ${ }^{0}$ particles similar to those observed in space-weathered lunar soils was achieved by direct mineral or glass reduction (Allen et al., 1993), short duration laser irradiation (e.g. Sasaki et al., 2001; 2002; 2003; Brunetto et al., 2005; Lazzarin et al., 2006; M arkley et al., 2013), ion bombardment (e.g. Marchi et al.; 2005, Lazzarin et al.; 2006), direct mineral / glass synthesis (Liu et al, 2007), or microwave irradiation (Tang et al., 2012) on various silicate minerals, meteorites or lunar rocks. Since then, laser irradiation together with ion bombardment 
has become a common laboratory tool for space weathering simulation, capable of reproducing most spectral changes. However, these methods do not provide sufficient control over npFe ${ }^{0}$ particle size and concentration and, thus, do not enable quantitative space weathering simulations.

Only a handful of studies were devoted to control the size and quantity of $n p \mathrm{Fe}^{0}$ and to verify its effect on spectral properties. For example, Allen et al. (1996) produced $6 \mathrm{~nm}$ sized npFe ${ }^{0}$ particles on a silica gel substrate with $6 \mathrm{~nm}$ pores through impregnating the pores with ferric nitrate solution and subsequent reduction of iron in a hydrogen atmosphere. Noble et al. (2007) enhanced this technique with silica gel substrates featuring pore sizes of $2.3,6,25$ and $50 \mathrm{~nm}$ resulting in a better control of $\mathrm{npFe}^{0}$ size and concentration. The changes in optical properties of such treated samples resembled the space weathering effects observed in lunar soils and it was possible to evaluate the influence of $n \mathrm{nFe}^{0}$ particle size on spectral red slope.

In this work we present a new method of controlled $\mathrm{npFe}^{0}$ production on surface of olivine grains. We also evaluate the influence of $\mathrm{npFe}^{0}$ concentration and particle-size on olivine spectral darkening and reddening, as well as intensity reduction, shape, and position of the olivine $1 \mu \mathrm{m}$ absorption band.

\section{Materials and methods}

Samples

Natural olivine from Åneim, Norway was used in this study. A pale green, polycrystalline olivine sample was thin-sectioned, and analyzed using a CAM ECA SX-100 electron microprobe (Table 1). Another part of the sample was crushed and inclusion-free fragments were hand-picked using an optical microscope. Subsequently, the olivine grains were pulverized in an agate mortar and sieved 
to obtain fine $(10-80 \mu \mathrm{m})$ powder. Part of the powder was preserved for reference measurements while the rest was thermally treated in a two-step heating method to achieve the formation of npFe ${ }^{0}$ on the surface of olivine grains.

npFe ${ }^{0}$ production and characterization

A two-step thermal treatment method was developed to produce $n p F e^{0}$ on the surface of olivine grains. Furthermore, $n p \mathrm{Fe}^{0}$ abundance and particle size control was achieved through variations in temperature and duration of the treatment steps.

In the first step, the olivine powder was heated in air (under oxidizing condition) using a Linn LM 112.07 muffle furnace. Various temperatures and heating durations were tested (Table 2 ) in order to induce partial oxidation of the iron ions that were liberated from the olivine structure, primarily those close to the surface of the olivine grains. The temperatures were selected to be low enough to preserve the olivine structure undamaged (e.g. Barcova et al., 2003; M ichel et al., 2013). The oxidation of $\mathrm{Fe}^{2+}$ ions leads to a charge imbalance in the olivine structure causing diffusion of $\mathrm{Fe}^{3+}$ atoms to the surface where they precipitate in the form of $\mathrm{Fe}^{3+}$ oxide nanoparticles and create vacancies in the olivine crystal structure (Zboril et al., 2003; Barcova et al., 2003).

In the second step, the $\mathrm{Fe}^{3+}$ oxide nanoparticles were reduced into $\mathrm{npFe}^{0}$ in a hydrogen atmosphere (using a steady hydrogen flow at $500^{\circ} \mathrm{C}$ for 1 hour with an Anton Paar XRK900 reactor). The reduction process was monitored in-situ using X-ray powder diffraction (XRD). The freshly produced npFe ${ }^{0}$ particles were surface-passivated for 30 minutes in $30^{\circ} \mathrm{C}$ gas mixture flow of $\mathrm{N}_{2}$ with $2 \% \mathrm{O}_{2}$ to minimize oxidation upon contact with ambient air.

The XRD was performed using a PANalytical X'Pert PRO MPD diffractometer (iron-filtered CoK ${ }_{\alpha}$ radiation: $\lambda=0.178901 \mathrm{~nm}, 40 \mathrm{kV}$ and $30 \mathrm{~mA}$ ) in the Bragg-Brentano geometry and equipped with an X'Celerator detector, that has programmable divergence and diffracted beam anti-scatter slits. 
The samples were placed into a shallow cavity sample holder (made of Macor) and repeatedlyscanned in the $2 \theta$ range of $5-120^{\circ}$ (resolution of $0.017^{\circ} 2 \theta$ ) at specific temperatures. SRM 640 (Si) and SRM $660\left(\mathrm{LaB}_{6}\right)$ commercial standards from NIST (National Institute of Standards and Technology) were used for evaluation of the line positions and instrumental line broadening, respectively. Identification of crystalline phases was performed using the HighScore Plus (PANalytical) software in conjunction with the PDF-4+database.

The size and concentration of $\mathrm{npFe}^{0}$ was determined using a combination of transmission electron microscopy (TEM), frequency dependence of magnetic susceptibility, and magnetic hysteresis measurements, Composition of the $\mathrm{npFe}^{0}$ was verified using scanning transmission electron microscopy equipped with energy-dispersive X-ray spectroscopy (STEM-EDX).

The TEM observations and STEM -EDX analysis was done using a Teknai F30 TEM/STEM with an EDAX Si/Li EDX spectrometer. The TEM observations of npFe ${ }^{0}$ particles were done on sharp, thin edges of olivine powder grains placed on a copper grid holder.

The frequency dependence of magnetic susceptibility was tested using a ZH instruments SM100/105 susceptibility meters at 16, 32, 64, 128 and $256 \mathrm{kHz}$ frequency steps and $160 \mathrm{~A} / \mathrm{m}$ RM S field intensity.

The magnetic hysteresis measurements were accomplished using a Princeton Measurements Micromag Model 3900 VSM (Vibrating Sample Magnetometer). All magnetizations were normalized by sample. First, a hysteresis loop of the olivine precursor was measured. This loop was subsequently used for background subtraction (to subtract paramagnetic/diamagnetic contribution of olivine and sample holder) of all thermally-treated olivine sample hysteresis loops. After background subtraction, any residual paramagnetic/diamagnetic slope was removed. Resulting hysteresis loops correspond to $\mathrm{npFe}^{0}$ produced in our samples. The concentration of 
$\mathrm{npFe} \mathrm{e}^{0}$ can be estimated by comparison of the sample saturation magnetization to that of a pure metallic iron (218 Am²/kg, Dunlop and Özdemir, 2001, p. 51).

For room-temperature ${ }^{57}$ Fe M össbauer measurements, a M össbauer spectrometer with a ${ }^{57} \mathrm{Co}(\mathrm{Rh})$ source of $y$-rays was used. The values of the derived hyperfine Mössbauer parameters are attributed to metallic iron ( $\alpha-\mathrm{Fe}$ ) at room temperature. Mössbauer spectra were fitted by means of the Lorentzian line shapes using the least squares method featured in the MossWinn analysis program. To obtain a qualitatively resolved Mössbauer spectrum for low npFe ${ }^{0}$ concentration, a long measurement time was applied (22 days).

The spectral measurements of fresh and modified olivine samples were done in visible - near infrared (VIS-NIR) range of 350-2400 nm using an Analytical Spectral Devices FieldSpec Pro spectrometer calibrated with a Labsphere SRS-99-020 Spectralon white standard. The reflectance is determined at $550 \mathrm{~nm}$. The spectral slope is calculated as the difference in normalized reflectance at $1689 \mathrm{~nm}$ and $630 \mathrm{~nm}$ divided by $1059 \mathrm{~nm}$. The normalized reflectance was calculated as reflectance divided by reflectance at $550 \mathrm{~nm}$. The $1 \mu \mathrm{m}$ absorption band depth is calculated as the normalized average reflectance at 630 and $1689 \mathrm{~nm}$ minus the band minimum. The $1 \mu \mathrm{m}$ absorption band minimum position was manually read from the data files. To further study shift of the band minimum in our samples the modified Gaussian model (M GM, Sunshine et al., 1999, MATLAB code available on RELAB web page http://www .planetary.brown.edu/mgm/index.html) was applied to the spectral data.

\section{Results}

The combination of the two successive thermal treatment steps resulted in a formation of $n p F e^{0}$ ( $\alpha$-Fe, bcc structure) on the olivine grains with a well-controlled particle size and distribution over the surface. The characteristics of such npFe $e^{0}$ particles is comparable to the $\mathrm{npFe}^{0}$ observed in 
space weathered extraterrestrial materials. Table 3 summarizes spectral properties of the thermally-treated olivine samples and $\mathrm{npFe}^{0}$ concentration estimate derived from magnetic hysteresis measurements. There is a quasi-exponential trend in the $n p F e^{0}$ concentration with increasing temperature of the first heating step (Fig. 1). Longer duration of the first heating step also increases the $n p \mathrm{Fe}^{0}$ concentration. Due to the quasi-exponential trend between $n p F e^{0}$ concentration and first step heating temperature, the samples heated to temperatures in excess of $750^{\circ} \mathrm{C}$ become very rich in $\mathrm{npFe} \mathrm{e}^{0}$ and their reflectance spectra become very low. Thus, in order to reduce the amount of $n p F e^{0}$ in the $850^{\circ} \mathrm{C}$ sample to the level of other samples, the duration of the first heating step was reduced to 30 seconds.

The size of the $n p F e^{0}$ particles in all samples as seen by TEM (Fig. 2) is in $\sim 5-20 \mathrm{~nm}$ range with the majority ( $-90 \%)$ being in the $7-15 \mathrm{~nm}$ range. An exception is the sample heated to $850^{\circ} \mathrm{C}(850 \mathrm{~s} 30)$ where an additional population of larger $\sim 40-50 \mathrm{~nm} n p F e^{0}$ particles can be observed (Fig. 3). The larger particles most likely formed by sintering of smaller particles into clusters.

The above mentioned $n p F e^{0}$ particle range is supported by a frequency dependence of the magnetic susceptibility data which occurs in particles that are in a superparamagnetic (SP) state that requires iron particles smaller than $\sim 8 \mathrm{~nm}$ (Kneller and Luborsky, 1963; Dunlop and Özdemir, 2001, p. 131). No detectable systematic frequency dependence was observed in any sample, indicating that majority of the $\mathrm{npFe} \mathrm{e}^{0}$ particles were larger than the $-8 \mathrm{~nm}$ threshold.

One single $n p F e^{0}$ particle was investigated by high-resolution TEM in order to determine the lattice spacing on the single nanoparticle. Lattice fringes of $0.19 \pm 0.05 \mathrm{~nm}$ were observed (Fig. 4) which is consistent with the spacing of (110) of $\alpha-F e d_{110}=0.203 \mathrm{~nm}$ (Noguchi et al., 2011). The surface passivation of $\mathrm{npFe}^{0}$ on the olivine limited the formation of iron oxides to a thin shell on the metallic nanoparticle core (cf. Filip et al., 2007 and Siskova et al., 2012). This is well 
documented on STEM-EDX data as little oxygen (mostly originating from olivine background) is detected in individual npFe ${ }^{0}$ particles (Fig. 5).

Additionally, the modified olivine samples were studied by means of zero-field ${ }^{57} \mathrm{Fe}$ Mössbauer spectroscopy at room temperature. Only paramagnetic doublet (isomer shift $\delta=1.12 \mathrm{~mm} / \mathrm{s}$ and quadrupole split $\left.\varepsilon_{\mathrm{Q}}=2.96 \mathrm{~mm} / \mathrm{s}\right)$ and the singlet $(\delta=0.53 \mathrm{~mm} / \mathrm{s})$, both originating from $\mathrm{Fe}^{2+}$ in the two olivine octahedral sites with different point symmetries, are visible in the spectra. The ferromagnetic sextet component corresponding to ferromagnetic metallic iron is not observed due to $\mathrm{npFe}^{0}$ amount being below $\mathrm{M}$ össbauer spectroscopy resolution.

The reflectance spectra of fresh and modified olivine samples were measured in 350-2400 nm (VIS-NIR) range. A progressive trend in the reflectance reduction (darkening), in $1 \mu \mathrm{m}$ absorption band depth reduction, slope change (reddening), shift in $1 \mu \mathrm{m}$ band center position, and $1 \mu \mathrm{m}$ band width at half depth is observed with the increasing npFe ${ }^{0}$ concentration (Fig. 6).

\section{Discussion}

The size range of the $n p F e^{0}(\sim 5-20 \mathrm{~nm})$ produced in olivine powders by our method is slightly larger than that found in vapor deposition rims $(\sim 3 \mathrm{~nm})$, but considerably smaller than the nanophase iron found in agglutinates (e.g. Pieters et al., 2000; Hapke, 2001, Keller and Clemett, 2001). The presence of the $\mathrm{npFe}^{0}$ is also seen in the strong alteration of the VIS-NIR spectra. Compared to spectra of the fresh olivine, samples with artificially produced $n p F e^{0}$ show spectral changes similar to these seen in naturally space-weathered lunar soils and asteroid surfaces (e.g. Hapke, 2001; Chapman, 2004). This gives us a confidence that despite slight differences between our samples and natural space weathering products (npFe ${ }^{0}$ particles on mineral grain surfaces vs. within coating rims, possible presence of a thin oxide shell on our npFe ${ }^{0}$ particles) our laboratory simulations are good proxy for natural space weathering and closely resemble optical effects of 
the fine nanophase iron fraction including slope change (Noble et al., 2007). The changes in olivine spectral parameters are quantified in Table 3. Because the $n \mathrm{nFe}^{0}$ particle size is kept constant (with exception of 850s30 sample), the spectral changes can be studied as a function of increasing $n p F e^{0}$ concentration. The results indicate that there is a linear trend between amount of $n p F e^{0}$ and the $1 \mu \mathrm{m}$ band center position (Fig. 7) and a logarithmic trend between the amount of npFe ${ }^{0}$ and the reflectance (Fig. 8), $1 \mu \mathrm{m}$ absorption band depth (Fig. 8), spectral slope (Fig. 9) and the $1 \mu \mathrm{m}$ band width at half depth (Fig. 10).

The linear trend between amount of $\mathrm{npFe}^{0}$ and the $1 \mu \mathrm{m}$ band center position is in contrast with results of previous studies (Hiroi and Sasaki, 2001; Sasaki et al., 2002; 2003; Brunetto et al,. 2005) where no significant changes of the band position were observed during simulated space weathering. However, compared to these studies, our samples cover a broader range of npFe ${ }^{0}$ amount and related spectral changes. It is apparent that for samples with less npFe ${ }^{0}$ (below 0.01 $w t \%$ and spectral changes of a similar magnitude as in above-mentioned studies) there is no significant change in band position. However, with higher $\mathrm{npFe}^{0}$ the amount of the shift in $1 \mu \mathrm{m}$ band center became more apparent. The mechanism behind the band shift may be $\mathrm{Fe}^{2+}$ disassociation from olivine structure into $n p F e^{0}$ particles. This is also supported by the linear correlation between $1 \mu \mathrm{m}$ band center and amount of $\mathrm{npFe}^{0}$. The possible occurrence of this process in extremely weathered natural planetary surfaces is uncertain and requires further studies. In general, asteroidal regoliths do not get as mature as lunar regolith and olivine is minor constituent of the lunar regolith.

The band center shift was further studied using the MGM model. As the olivine $1 \mu \mathrm{m}$ absorption band is a superposition of three individual bands related to the different positions of $\mathrm{Fe}^{2+}$ ions in the olivine crystal structure (Burns, 1970), three Gaussian bands were used in the M GM model. The results are summarized in table 4 and figure 11. MGM method gives reliable results for 
samples with small to moderate npFe $\mathrm{e}^{0}$ amounts up to sample $650 \mathrm{~h} 1$ with 0.023 wt $\%$ metallic Fe. (With higher npFe ${ }^{0}$ content the lower $1 \mu \mathrm{m}$ band intensity to noise ratio and significant red slope causes unreliable Gaussian modeling of olivine samples.) The MGM results indicate that the shift in $1 \mu \mathrm{m}$ band is primarily driven by changes in the third Gaussian band centered around $1200 \mathrm{~nm}$ (Fig. 11) which is caused (together with the $\sim 840 \mathrm{~nm}$ band) by the $\mathrm{Fe}^{2+}$ in $\mathrm{M}$ (2) position (e.g. Burns, 1970). The M GM model figures are available in on-line supplementary material (Figs. S1-S7).

The observed logarithmic trend between slope change and amount of $n p F e^{0}$ provides further insight into natural space weathering. A logarithmic trend between spectral slope change and the space weathering duration has been observed by Nesvorný et al. (2005) and Vernazza et al. (2009) for S-type asteroid families. In combination with our observation of a logarithmic trend between amount of $n p F e^{0}$ and reflectance, $1 \mu \mathrm{m}$ absorption band depth, and spectral slope, we have demonstrated four additional characteristics of space weathering: A similar logarithmic trend is valid between space weathering duration and (1) darkening, (2) reduction of $1 \mu \mathrm{m}$ olivine absorption band, and (3) $1 \mu \mathrm{m}$ band width at half depth. However, (4) the amount of npFe ${ }^{0}$ increases linearly with the duration of the space weathering.

The 850 s30 sample contains additional population of $-40-50 \mathrm{~nm} n p F e^{0}$ particles which accounts for its VIS-NIR spectral properties. The spectrum of this sample follows the trends observed for other samples. However, sample 850s30 does not fully follow the increasing red slope trend (Fig. 9). This is most likely due to the fact that part of its $n p F e^{0}$ is in a form of larger particles, which does not contribute to the red slope. This sample is analogous to lunar soils containing both small $n p F e^{0}$ fraction in vapor deposition rims as well as larger $n p F e^{0}$ particles in agglutinates. The relatively reduced magnitude of the slope change in this sample is in agreement with Noble et al. (2007) and Lucey and Noble (2008) who observed insignificant slope change for npFe ${ }^{0}$ particles larger than $\sim 50 \mathrm{~nm}$. 


\section{Conclusions}

The two-step thermal treatment method allows for controlled growth of iron nanoparticles on the surfaces of olivine powder grains. This enables quantitative investigations of the role of $\mathrm{npFe}^{0}$ in space weathering and related changes in reflectance spectra. Compared to fresh olivine, our olivine samples with artificially introduced $-5-20 \mathrm{~nm}$ sized $\mathrm{npFe}{ }^{0}$ particles exhibit the spectral characteristics of lunar-type space weathering. From a quantitative point of view, a linear trend is observed between the amount of $\mathrm{npFe}^{0}$ and $1 \mu \mathrm{m}$ band center position. This trend is more pronounced for samples with npFe ${ }^{0}$ amounts in excess of $0.015 \mathrm{wt} \%$. The mechanism behind the band shift may be $\mathrm{Fe}^{2+}$ disassociation from olivine structure into $\mathrm{npFe}^{0}$ particles.

A logarithmic trend is observed between amount of $\mathrm{npFe}^{0}$ and darkening, reduction of $1 \mu \mathrm{m}$ olivine absorption band, reddening, and $1 \mu \mathrm{m}$ band width at half depth. Observations of asteroid families show a logarithmic weathering trend between slope change and duration. Our results reveal four additional characteristics of space weathering: The logarithmic trend with space weathering duration is also valid for (1) darkening, (2) reduction of $1 \mu \mathrm{m}$ olivine absorption band, and (3) $1 \mu \mathrm{m}$ band width at half depth, while (4) the amount of $n \mathrm{nFe}^{0}$ increases linearly with duration.

The olivine sample with an additional population of larger $\mathrm{npFe}^{0}$ particles follows similar spectral trends as other samples, except for the reddening trend. This is interpreted as the larger, $(\sim 40$ $50 \mathrm{~nm}$ sized), $\mathrm{npFe}^{0}$ particles do not contribute to the slope change as efficiently as the smaller $n p F e^{0}$ fraction. 


\section{Acknowledgements}

The work was supported by M inistry of Education, Youth and Sports of the Czech Republic (grant no. LH12079, LK21303, MSM 0021620855), Academy of Finland (grant no. 257487), Czech Science Foundation (grant no. GACR P108/11/1350) and Palacký University, Olomouc, Czech Republic (grant no. PrF_2013_014). We also thank for the support by the Operational Program Research and Development for Innovations - European Regional Development Fund (CZ.1.05/2.1.00/03.0058) and Operational Program Education for Competitiveness - European Social Fund (CZ.1.07/2.3.00/20.0017, CZ.1.07/2.3.00/20.0170, CZ.1.07/2.3.00/20.0155, and CZ1.07/2.3.00/20.0056) of the M inistry of Education, Youth and Sports of the Czech Republic. This work (publication SSERVI-2014-080) was directly supported by NASA's Solar System Exploration Research Virtual Institute cooperative agreement notice NNA14AB05A.

\section{References}

Adams, J.B., Jones, R.L., 1970. Spectral reflectivity of lunar samples. Science 167, 737-739.

Adams, J.B., McCord, T.B., 1971. Alteration of lunar optical properties: age and composition effects. Science 171, 567-571.

Adams, J.B., McCord, T.B., 1973. Vitrification darkening in the lunar highlands and identification of Descartes material at the Apollo 16 sites. Proceedings of the Lunar and Planetary Science Conference 4, 163-177.

Allen, C.C., Lauer, H.V., Jr., M orris, R.V., and M cKay, D.S., 1993, Microscopic iron metal on glass and minerals - - a tool for studying regolith maturity. Icarus 104, 291-300.

Allen, C.C., Morris, R.V., McKay, D.S., 1996. An experimental analog to maturing lunar soil. Proceedings of the Lunar and Planetary Science Conference 37, 13-14. 
Barcova, K., Mashlan, M., Zboril, R., Martinec, P., 2003. Mossbauer study of transformation mechanism of Fe cations in olivine after thermal treatments in air. Journal of Radioanalytical and Nuclear Chemistry 255, 529-533. doi: 10.1023/A:1022588500878

Binzel, R.P., Morbidelli, A., Merouane, S., DeM eo, F.E., Birlan, M., Vernazza, P., Thomas, C.A., Rivkin, A.S., Bus, S.J., Tokunaga, A.T., 2010. Earth encounters as the origin of fresh surfaces on near-Earth asteroids. Nature 463, 331-334. doi:10.1038/nature08709.

Brunetto, R., Romano, F., Blanco, A., Fonti, S., Martino, M., Orofino, V., Verrienti, C., 2005. Space weathering of silicates simulated by nanosecond pulse UV excimer laser. Icarus 180, 546554. doi:10.1016/j.icarus.2005.10.016.

Burns, R.G., 1970. Crystal field spectra and evidence of cation ordering in olivine minerals. American M ineralogist 55, 1608-1632.

Chapman, C.R., 2004. Space weathering of asteroid surfaces. Annual Reviews 32, 539-567. doi:10.1016/j.icarus.2010.05.006.

Clark, B.E., Lucey, P., Helfenstein, P, Bell lii, J.F., Peterson, C., Veverka, J., M cconnochie, T., Robinson, M.S., Bussey, B., Murchie, S.L., Izenberg, N.I., Chapman, C.R., 2001. Space weathering on Eros: Constraints from albedo and spectral measurements of Psyche crater. M eteoritics and Planetary Science 36, 1617-1637. doi: 10.1111/j.1945-5100.2001.tb01853.x. Dunlop, D.J., Özdemir, Ö., 2001. Rock Magnetism: fundamentals and frontiers, Cambridge University Press, Cambridge, 573 pages.

Filip, J., Zbořil, R., Schneeweiss, O., Zeman, J., Černík, M., Kvapil, P., Otyepka, M., 2007. Environmental applications of chemically-pure natural ferrihydrite. Environmental Science \& Technology 41, 4367-4374.

Gaffey, M.J., 2010 Space weathering and the interpretation of asteroid reflectance spectra. Icarus $209,564-574$. 
Hapke, B., 2001. Space weathering from Mercury to the asteroid belt. Journal of Geophysical Research 106, 10039-10073.

Hapke, B., Cassidy, W., Wells, E., 1975. Effects of vapour-phase deposition processes on the optical chemical and magnetic properties of the lunar regolith, Moon 13, 339-354.

Hiroi, T., Abe, M., Kitazato, K., Abe, S., Clark, B.E., Sasaki, S., Ishiguro, M., Barnouin-J ha, O.S., 2006. Developing space weathering on the Asteroid 25143 Itokawa. Nature 443, 56-58. doi:10.1038/nature05073.

Hiroi, T., Sasaki, S., 2001. Importance of space weathering simulation products in compositional modeling of asteroids: 349 Dembowska and 446 Aeternitas as examples. Meteoritics and Planetary Science 36, 1587-1596.

Keller, L.P., Clemett, S.J., 2001. Formation of nanophase iron in the lunar regolith. Lunar and Planetary Science Conference XXXII, abstract no. 2097.

Keller, L., M cKay, D., 1997. The nature and origin of rims on lunar soil grains. Geochimica et Cosmochimica Acta 61, 2331-2340.

Keller, L., McKay, D., 1993. Discovery of vapor deposits in the lunar regolith. Science, 261, 13051307. doi: $10.2307 / 2882155$

Kneller, E.F., Luborsky, F.E., 1963. Particle size dependence of coercivity and remanence of singledomain particles. Journal of Applied Physics 34, 656-658.

Lazzarin, M., M archi, S., M oroz, L.V., Brunetto, R., Magrin, S., Paolicchi, P., Strazzulla, G., 2006. Space Weathering in the Main Asteroid Belt: The Big Picture. The Astrophysical Journal Letters 647, L179-L182, doi: 10.1086/507448.

Liu, Y., Taylor, L.A., Thompson, J.R., Schnare, D.W., Park, J.S., 2007. Unique properties of lunar impact glass: Nanophase metallic Fe synthesis. American M ineralogist 92, 1420-1427. 
Lucey, P.G., Noble, S.K., 2008. Experimental test of a radiative transfer model of the optical effects of space weathering. Icarus 197, 348-353. doi: 10.1016/j.icarus.2008.05.008

Markley, M.M., Fuller, M.D., Kletetschka, G., 2013. Magnetic scanning of iron blebs in laser irradiated olivine grains. M eteoritics \& Planetary Science 48, A233.

Marchi, S., Brunetto, R., Magrin, S., Lazzarin, M., Gandolfi, D., 2005. Space weathering of nearEarth and main belt silicate-rich asteroids: observations and ion irradiation experiments. Astronomy \& Astrophysics 443, 769-775. doi: 10.1051/0004-6361:20053525

McCord, T.B., Johnson, T.V., 1970. Lunar spectral reflectivity $(0.30$ to 2.50 microns $)$ and implications for remote mineralogical analysis. Science 169, 855-858.

McCord, T.B., Adams, J.B., 1973. Progress in remote optical analysis of lunar surface composition. Moon 7, 453-474.

M ichel, R., Ammar, M.R., Poirier, J., Simon, P., 2013. Phase transformation characterization of olivine subjected to high temperature in air. Ceramics International 39, 5287-5294.

Nesvorný, D., Jedlicke, R., Whiteley, R.J., Ivezić, Ž., 2005. Evidence for asteroid space weathering from the Sloan Digital Sky Survey. Icarus 173, 132-152.doi: 10.1016/j.icarus.2004.07.026

Noble, S.K., Pieters, C.M., Keller, L.P., 2007. An experimental approach to understanding the optical effects of space weathering. Icarus 192, 629-642. doi:10.1016/j.icarus.2007.07.021.

Noguchi T., Nakamura T., Kimura M., et al., 2011. Incipient Space Weathering Observed on the Surface of Itokawa Dust Particles. Science 333. 1121-1125. doi: 10.1126/science.1207794.

Pieters, C.M., Taylor, L.A., Noble, S.K., Keller, L.P., Hapke, B., M orris, R.V., Allen, C.C., M cKay, D.S., Wentworth, S., 2000. Space weathering on airless bodies: Resolving a mystery with lunar samples. M eteoritics and Planetary Science 35, 1101-1107. 
Pieters, C.M., Fischer, E.M., Rode, O., Basu, A., 1993. Optical effects of space weathering: The role of the finest fraction. Journal of Geophysical Research: Planets 98, 20817-20824. doi: 10.1029/93JE02467

Sasaki, S., Kurahashi, E., Yamanaka, C., Nakamura, K., 2003. Laboratory simulation of space weathering: Changes of optical properties and TEM/ESR confirmation of nanophase metallic iron. Advances in Space Research 31, 2537-2542. doi: 10.1016/S0273-1177(03)00575-1

Sasaki, S, Hiroi, T., Nakamura, K., Hamabe, Y., Kurahashi, F., Yamada, M., 2002. Simulation of space weathering by nanosecond pulse laser heating: dependence on mineral composition, weathering trend of asteroids and discovery of nanophase iron particles. Advances in Space Research 29, 783-788.

Sasaki, S., Nakamura, K., Hamabe, Y., Kurahashi, E., Hiroi, T., 2001. Production of iron nanoparticles by laser irradiation in a simulation of lunar-like space weathering. Nature 410, 555-557. doi: $10.1038 / 35069013$

Siskova, K., Tucek, J., M achala, L., Otyepkova, E., Filip, J., Safarova, K., Pechousek, J., Zboril, R., 2012. Air-stable nZVI formation mediated by glutamic acid: solid-state storable material exhibiting 2D chain morphology and high reactivity in aqueous environment. Journal of Nanoparticle Research 14, 805 (13 pages). doi: 10.1007/s11051-012-0805-9

Sunshine, J.M ., Pieters, C.M., Pratt, S.F., M cNaron-Brown, K.S., 1999. Absorption Band M odeling in Reflectance Spectra: Availability of the Modified Gaussian Model. Lunar and Planetary Science Conference XXX, abstract no. 1306.

Tang, H., Wang, S., Li, X., 2012: Simulation of nanophase iron production in lunar space weathering. Planetary and Space Science 60, 322-327. doi: 10.1016/j.pss.2011.10.006 
Taylor, L.A., Pieters, C.M., M orris, R.V., Keller, L.P., M cKay, D.S., 2001. Lunar mare soils: Space weathering and the major effects of surface-correlated nanophase Fe. Journal of Geophysical Research: Planets 106, 27985-28000. doi: 10.1029/2000JE001402

Vernazza, P., Binzel, R.P., Rossi, A., Fulchignoni, M., Birlan, M., 2009. Solar wind as the origin of rapid reddening of asteroid surfaces. Nature 458, 993-995. doi:10.1038/nature07956

Zboril, R., Mashlan, M., Barcova, K., Walla, J., Ferrow, E., M artinec, P., 2003. Thermal behaviour of pyrope at 1000 and $1100^{\circ} \mathrm{C}$ : mechanism of $\mathrm{Fe}^{2+}$ oxidation and decomposition model. Physics and Chemistry of M inerals 30, 620-627. 
Table 1. Chemical analyses of olivine used in this study.

\begin{tabular}{|c|c|c|c|c|c|c|c|c|c|c|c|c|c|c|c|c|c|c|c|c|c|c|}
\hline & 1 & 2 & 3 & 4 & 5 & 6 & 7 & 8 & 9 & 10 & 11 & 12 & 13 & 14 & 15 & 16 & 17 & 18 & 19 & 20 & mean & s.d. \\
\hline \multicolumn{23}{|c|}{ Analyses in wt $\%$} \\
\hline $\mathrm{SiO}_{2}$ & 40.88 & 41.25 & 41.75 & 41.33 & 41.23 & 41.26 & 41.38 & 41.44 & 41.19 & 41.18 & 41.09 & 41.77 & 41.52 & 41.33 & 41.64 & 41.47 & 41.58 & 41.41 & 41.94 & 41.95 & 41.43 & 0.28 \\
\hline $\mathrm{Al}_{2} \mathrm{O}_{3}$ & 0.04 & b.d.l. & b.d.l. & b.d.l. & b.d.l. & b.d.l. & 0.04 & b.d.l. & b.d.l. & b.d.l. & b.d.l. & b.d.l. & b.d.l. & b.d.l. & b.d.l. & b.d.l. & b.d.l. & b.d.l. & b.d.l. & b.d.l. & 0.04 & 0.00 \\
\hline $\mathrm{FeO}^{\mathrm{T}}$ & 6.69 & 6.67 & 6.58 & 6.72 & 6.67 & 6.80 & 6.63 & 6.69 & 6.72 & 6.74 & 6.67 & 6.64 & 6.71 & 6.75 & 6.63 & 6.68 & 6.67 & 6.61 & 6.63 & 6.68 & 6.68 & 0.05 \\
\hline $\mathrm{NiO}$ & 0.39 & 0.37 & 0.41 & 0.39 & 0.36 & 0.38 & 0.38 & 0.38 & 0.38 & 0.39 & 0.37 & 0.40 & 0.39 & 0.37 & 0.38 & 0.39 & 0.38 & 0.39 & 0.38 & 0.38 & 0.38 & 0.01 \\
\hline $\mathrm{MnO}$ & 0.13 & 0.11 & b.d.l. & b.d.l. & 0.11 & 0.12 & b.d.l. & b.d.l. & 0.12 & b.d.l. & 0.15 & 0.12 & b.d.l. & b.d.l. & 0.10 & 0.13 & b.d.l. & b.d.l. & b.d.l. & b.d.l. & 0.12 & 0.01 \\
\hline $\mathrm{MgO}$ & 51.68 & 51.25 & 51.78 & 51.56 & 51.83 & 51.90 & 51.95 & 51.35 & 51.60 & 51.67 & 51.61 & 51.78 & 51.67 & 51.40 & 51.23 & 51.34 & 51.68 & 51.30 & 51.24 & 51.07 & 51.54 & 0.25 \\
\hline Total & 99.80 & 99.64 & 100.52 & 100.00 & 100.21 & 100.44 & 100.38 & 99.85 & 100.00 & 99.97 & 99.90 & 100.71 & 100.28 & 99.86 & 99.97 & 100.01 & 100.30 & 99.71 & 100.20 & 100.07 & 100.09 & 0.28 \\
\hline \multicolumn{23}{|c|}{ Coeficients of empirical formulae in a.p.f.u. calculated on the basis of 4 oxygens in formula unit } \\
\hline $\mathrm{Si}$ & 0.992 & 1.001 & 1.003 & 0.999 & 0.995 & 0.994 & 0.996 & 1.003 & 0.997 & 0.996 & 0.995 & 1.002 & 1.001 & 1.000 & 1.006 & 1.002 & 1.001 & 1.003 & 1.010 & 1.011 & 1.000 & 0.005 \\
\hline $\mathrm{Al}$ & 0.001 & & & & & & 0.001 & & & & & & & & & & & & & & 0.001 & \\
\hline $\mathrm{Fe}$ & 0.136 & 0.135 & 0.132 & 0.136 & 0.135 & 0.137 & 0.133 & 0.135 & 0.136 & 0.136 & 0.135 & 0.133 & 0.135 & 0.137 & 0.134 & 0.135 & 0.134 & 0.134 & 0.134 & 0.135 & 0.135 & 0.001 \\
\hline $\mathrm{Ni}$ & 0.008 & 0.007 & 0.008 & 0.008 & 0.007 & 0.007 & 0.007 & 0.007 & 0.007 & 0.008 & 0.007 & 0.008 & 0.008 & 0.007 & 0.007 & 0.008 & 0.007 & 0.008 & 0.007 & 0.007 & 0.007 & 0.000 \\
\hline $\mathrm{Mn}$ & 0.003 & 0.002 & & & 0.002 & 0.002 & & & 0.002 & & 0.003 & 0.002 & & & 0.002 & 0.003 & & & & & 0.002 & 0.000 \\
\hline $\mathrm{Mg}$ & 1.869 & 1.854 & 1.854 & 1.858 & 1.865 & 1.865 & 1.865 & 1.852 & 1.861 & 1.864 & 1.864 & 1.852 & 1.856 & 1.855 & 1.845 & 1.850 & 1.856 & 1.852 & 1.839 & 1.836 & 1.856 & 0.009 \\
\hline \multirow[t]{2}{*}{ Total } & 3.008 & 2.999 & 2.997 & 3.001 & 3.005 & 3.006 & 3.003 & 2.997 & 3.003 & 3.004 & 3.005 & 2.998 & 2.999 & 3.000 & 2.994 & 2.998 & 2.999 & 2.997 & 2.990 & 2.989 & 3.000 & 0.005 \\
\hline & & & & & & & & & & & & & & & & \multicolumn{7}{|c|}{ Forsterite content in mol. $\%$} \\
\hline Fo & 93.2 & 93.2 & 93.3 & 93.2 & 93.3 & 93.2 & 93.3 & 93.2 & 93.2 & 93.2 & 93.2 & 93.3 & 93.2 & 93.1 & 93.2 & 93.2 & 93.2 & 93.3 & 93.2 & 93.2 & 93.2 & 0.1 \\
\hline
\end{tabular}

Notes: a.p.f.u. - atom per formula unit; s.d. - standard deviation; b.d.I. indicates values below detection limit; $\mathrm{Ti}, \mathrm{Cr}$, $\mathrm{Ca}$, $\mathrm{Na}$, and $\mathrm{K}$ were found to be below detection limit in all analyses.

Measurement details: microprobe CAM ECA SX-100; accelerating voltage 15kV / sample current 10nA: $\mathrm{NaK}_{\alpha}$ (standard: jadeite; detection limit 550 ppm), $\mathrm{SiK}_{\alpha}$ (quartz; 480 ppm), M gK (diopside; 950 ppm), $\mathrm{KK}_{\alpha}$ (sanidine; 240 ppm), $\mathrm{CaK}_{\alpha}$ (diopside; 260 ppm), TiK (rutile; 320 ppm), AlK (jadeite; 330 ppm), $\mathrm{M} \mathrm{nK}_{\alpha}$ (rhodonite; 700 ppm), $\mathrm{CrK}_{\alpha}\left(\mathrm{Cr}_{2} \mathrm{O}_{3} ; 600\right.$ ppm); 20kV / 10nA: $\mathrm{FeK}_{\alpha}$ (magnetite; 450 ppm), NiK $($ NiSi; 230 ppm); beam diameter 2 $\mu \mathrm{m}$. 
Table 2. Conditions of sample processing. $T_{1}$ and $t_{1}$ are the temperature and time of the first heating step (in air). $T_{2}$ and $t_{2}$ are the temperature and time of the second heating step (in hydrogen). N/A - not applicable.

\begin{tabular}{|c|c|c|c|c|}
\hline Sample & $\mathrm{T}_{1}\left({ }^{\circ} \mathrm{C}\right)$ & $\mathrm{t}_{1}(\mathrm{~s})$ & $\mathrm{T}_{2}\left({ }^{\circ} \mathrm{C}\right)$ & $\mathrm{t}_{2}(\mathrm{~s})$ \\
\hline Raw olivine & $\mathrm{N} / \mathrm{A}$ & N/A & N/A & N/A \\
\hline 400h1 & 400 & 3600 & 500 & 3600 \\
\hline 450h1 & 450 & 3600 & 500 & 3600 \\
\hline 500h1 & 500 & 3600 & 500 & 3600 \\
\hline 550h1 & 550 & 3600 & 500 & 3600 \\
\hline $600 \mathrm{~h} 1$ & 600 & 3600 & 500 & 3600 \\
\hline 650h1 & 650 & 3600 & 500 & 3600 \\
\hline 700h1 & 700 & 3600 & 500 & 3600 \\
\hline 750h1 & 750 & 3600 & 500 & 3600 \\
\hline $850 s 30$ & 850 & 30 & 500 & 3600 \\
\hline
\end{tabular}


Table 3. Overview of sample spectral parameters, saturation magnetization $\left(\mathrm{J}_{\mathrm{s}}\right)$ and calculated $n p F e^{0}$ concentration. Iron concentration is calculated as the sample saturation magnetization divided by the saturation magnetization of pure iron $\left(218 \mathrm{Am}^{2} / \mathrm{kg}\right)$. * not possible to reliably determine.

$\begin{array}{lcccccrr}\text { Sample } & \begin{array}{c}\text { Slope } \\ \left(\mu \mathrm{m}^{-1}\right)\end{array} & \begin{array}{c}1 \mu \mathrm{m} \\ \text { depth }\end{array} & \begin{array}{c}\text { Albedo } \\ \text { at } 550 \\ \mathrm{~nm}\end{array} & \begin{array}{c}\text { 1um } \\ \text { band } \\ \text { center } \\ (\mathrm{nm})\end{array} & \begin{array}{c}1 \mu \mathrm{m} \\ \text { band } \\ \text { width at } \\ \text { half } \\ \text { depth } \\ (\mathrm{nm})\end{array} & \begin{array}{c}\mathrm{J}_{\mathrm{s}} \\ \left(\mathrm{mAm}^{2} / \mathrm{kg}\right)\end{array} & \begin{array}{c}\mathrm{Fe} \\ (\mathrm{wt} \%)\end{array} \\ \text { Olivine } & 0.031 & 0.21 & 0.78 & 1053 & 466 & 0 & 0.0 \\ \text { 400h1 } & 0.15 & 0.18 & 0.61 & 1048 & 356 & 16.44 & 0.0075 \\ \text { 450h1 } & 0.17 & 0.18 & 0.60 & 1048 & 347 & 18.43 & 0.0085 \\ \text { 500h1 } & 0.25 & 0.20 & 0.42 & 1048 & 306 & 24.59 & 0.011 \\ \text { 550h1 } & 0.22 & 0.15 & 0.52 & 1046 & 265 & 27.78 & 0.013 \\ \text { 600h1 } & 0.36 & 0.16 & 0.34 & 1045 & 222 & 31.89 & 0.015 \\ \text { 650h1 } & 0.41 & 0.10 & 0.28 & 1041 & 165 & 49.71 & 0.023 \\ 700 \mathrm{~h} 1 & 0.57 & 0.026 & 0.16 & 1031 & 118 & 128.7 & 0.059 \\ 750 \mathrm{~h} 1 & 0.69 & 0.00 & 0.11 & 970 & * & 253.2 & 0.12 \\ \text { 850s30 } & 0.36 & 0.034 & 0.28 & 1034 & 139 & 106.3 & 0.049\end{array}$


Table 4. Modified Gaussian model parameters of the olivine absorption band. C1-3 are the three Gaussian centers. FWHM 1-3 are the full widths at half maximum. S1-3 are the Gaussian strengths. RMSD is the root mean square deviation.

\begin{tabular}{|c|c|c|c|c|c|c|c|c|c|c|}
\hline & $\mathrm{Cl}(\mathrm{nm})$ & C2 (nm) & C3 (nm) & $\begin{array}{l}\text { FWHM } 1 \\
(\mathrm{~nm})\end{array}$ & $\begin{array}{l}\text { FWHM } 2 \\
(\mathrm{~nm})\end{array}$ & $\begin{array}{l}\text { FWHN } \\
(\mathrm{nm})\end{array}$ & S1 & S2 & S3 & RM SD \\
\hline $\begin{array}{l}\text { Raw } \\
\text { olivine }\end{array}$ & 826 & 1022 & 1229 & 124 & 221 & 465 & -0.0863 & -0.239 & -0.276 & 2.27E-02 \\
\hline $400 \mathrm{~h} 1$ & 837 & 1026 & 1202 & 170 & 181 & 436 & -0.093 & -0.195 & -0.265 & 4.73E-03 \\
\hline $450 \mathrm{~h} 1$ & 834 & 1025 & 1201 & 166 & 184 & 450 & -0.0916 & -0.192 & -0.27 & $7.28 \mathrm{E}-03$ \\
\hline $500 \mathrm{~h} 1$ & 847 & 1032 & 1194 & 214 & 169 & 442 & -0.126 & -0.188 & -0.303 & $3.77 \mathrm{E}-03$ \\
\hline $550 \mathrm{~h} 1$ & 835 & 1028 & 1195 & 175 & 180 & 454 & -0.0821 & -0.161 & -0.237 & $5.88 \mathrm{E}-03$ \\
\hline $600 \mathrm{~h} 1$ & 853 & 1039 & 1187 & 265 & 160 & 464 & -0.104 & -0.134 & -0.277 & $2.86 \mathrm{E}-03$ \\
\hline $50 \mathrm{~h} 1$ & 846 & 1040 & 1171 & 280 & 154 & 487 & -0.0697 & -0.101 & -0.226 & $2.77 \mathrm{E}-03$ \\
\hline
\end{tabular}


Fig. 1. The relation between the temperature of first heating step (in air) and amount of iron produced in form of iron nanoparticles. The heating duration was 1 hour for all displayed samples.

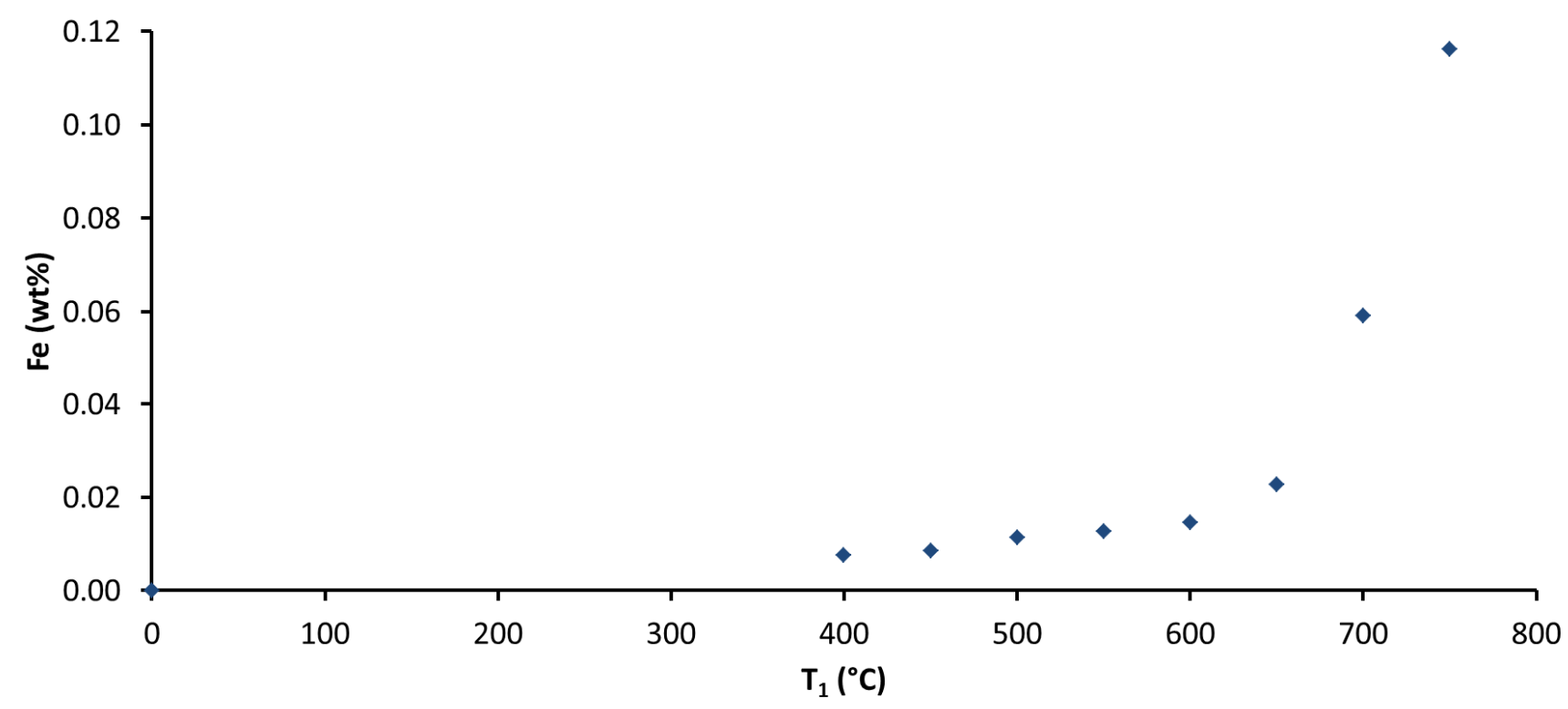


Fig. 2. TEM image of $\sim 5-20 \mathrm{~nm}$ sized nanoparticles on olivine powder grains (sample $600 \mathrm{~h} 1$ ).

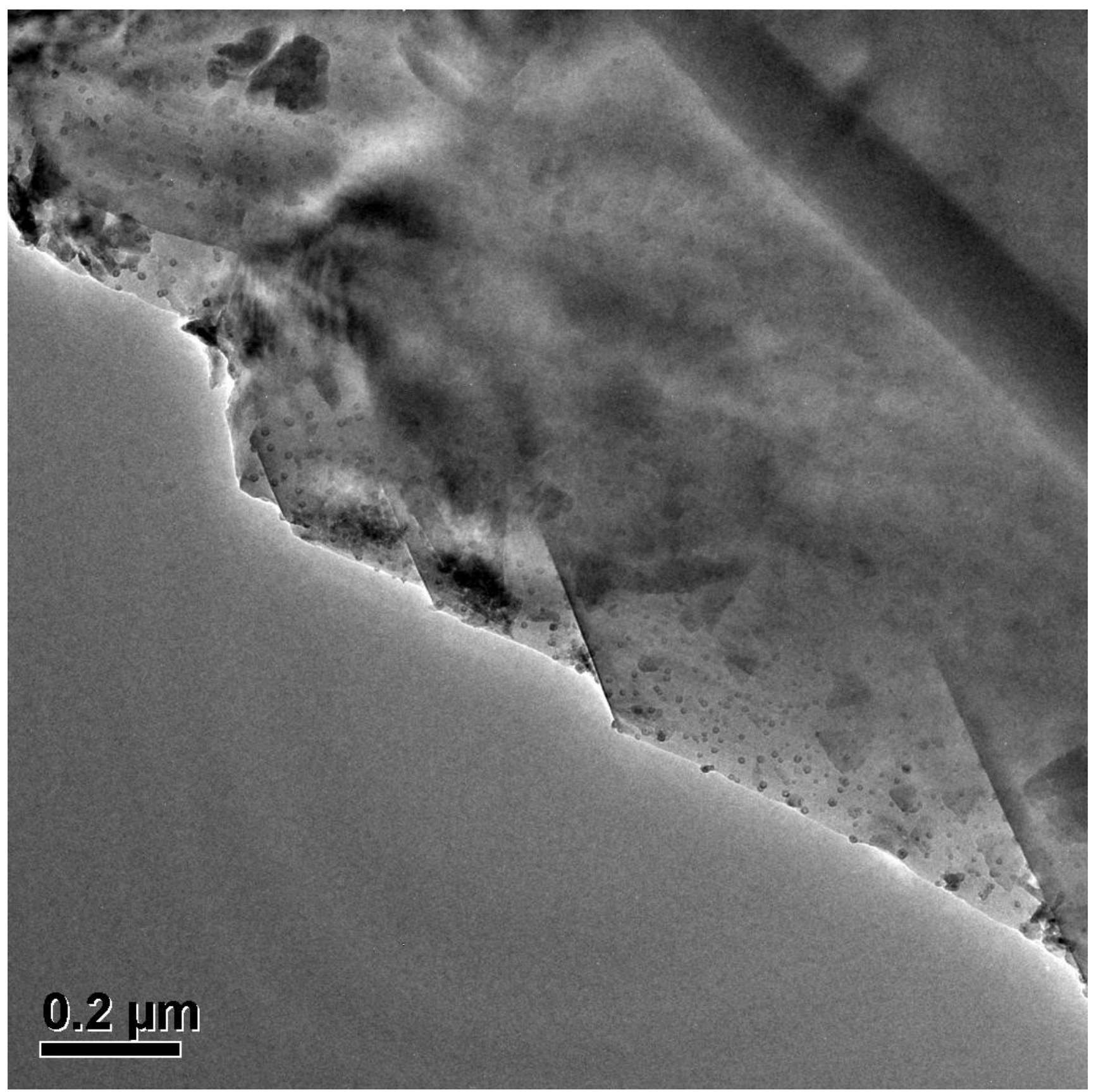


Fig. 3. TEM image of nanoparticles on olivine powder grains of the 850 s30 sample. Two populations can be observed ( $-5-20 \mathrm{~nm}$ and $\sim 40-50 \mathrm{~nm})$. 
Fig. 4. High resolution TEM image of a single iron nanoparticle. The lattice fringes are highlighted by yellow circle. The lattice spacing is $0.19 \pm 0.05 \mathrm{~nm}$.

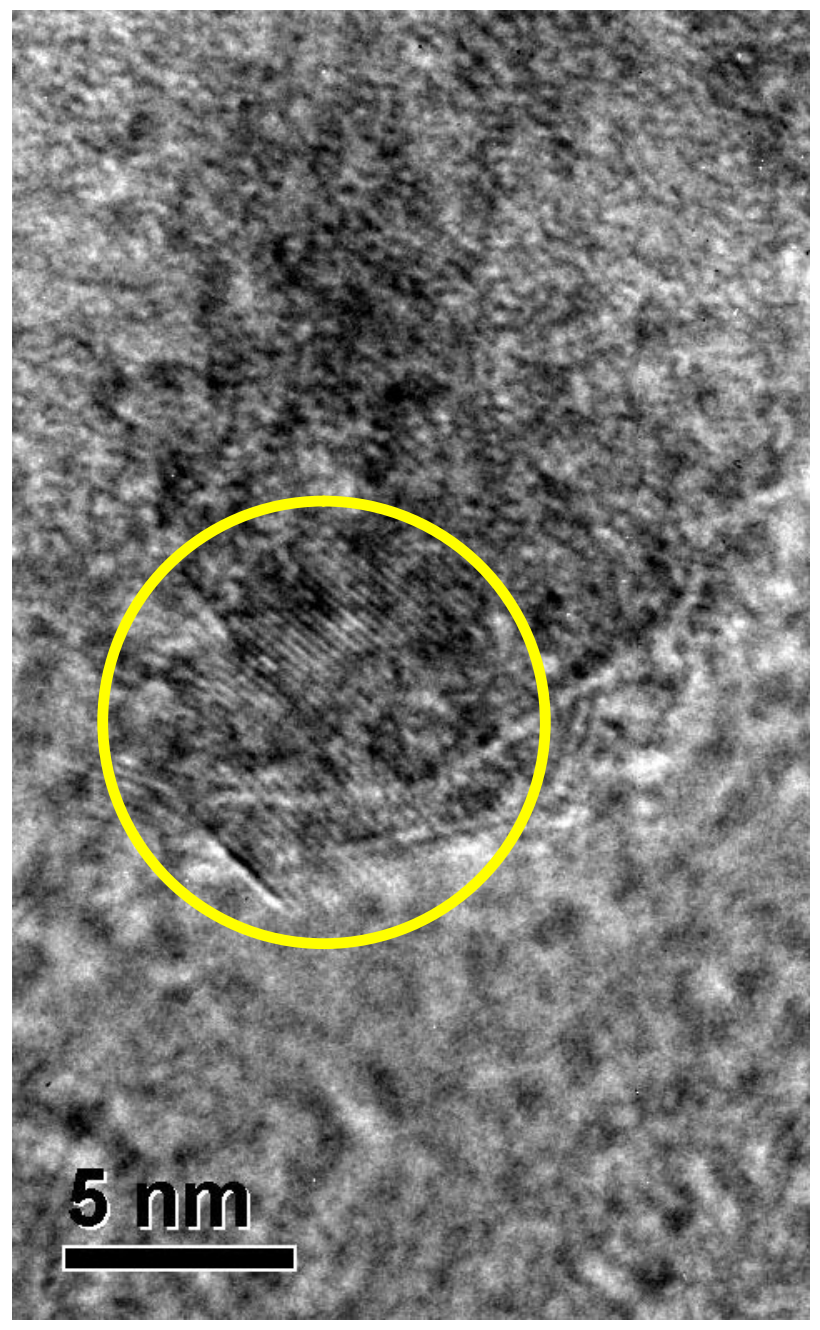


Fig. 5. STEM EDX spectra of an individual iron nanoparticle. These data show that iron nanoparticle oxidation was kept to minimum since only a minor peak of oxygen is observed and that is most likely caused by the olivine background (also indicated by a minor peak of silica and magnesium). Peak of copper is the signal of the sample holder.

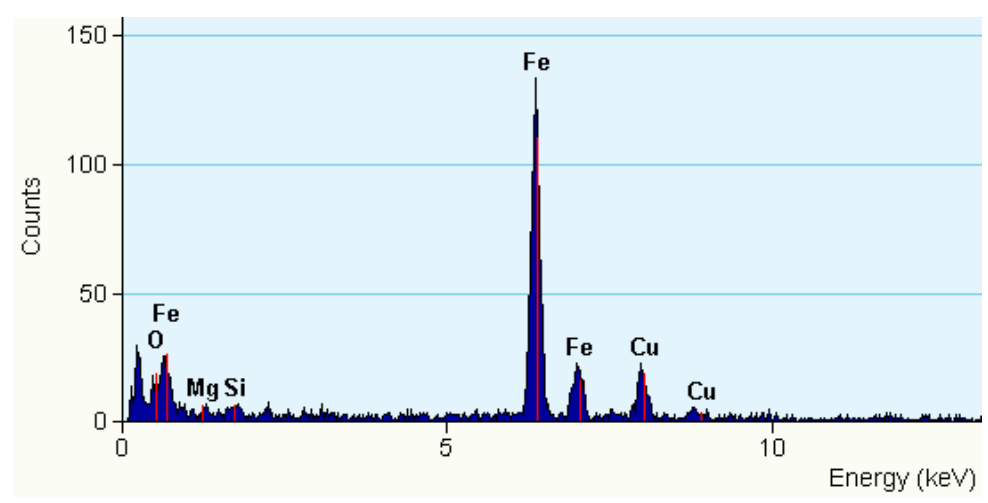


Fig. 6. VIS-NIR reflectance spectra (up - absolute values, down - normalized at $550 \mathrm{~nm}$ ) of the fresh and modified olivine samples with increasing npFe ${ }^{0}$ concentration (in wt $\%$ ) as determined from the saturation magnetization. All samples show the continuous trend of $1 \mu \mathrm{m}$ olivine absorption band reduction and increasing red slope with increasing amount of the $\mathrm{npFe}^{0}$. The $850^{\circ} \mathrm{C}$ sample does not follow the increasing red slope trend because it contains additional larger $n p F e^{0}$ particles that do not contribute to the red slope.
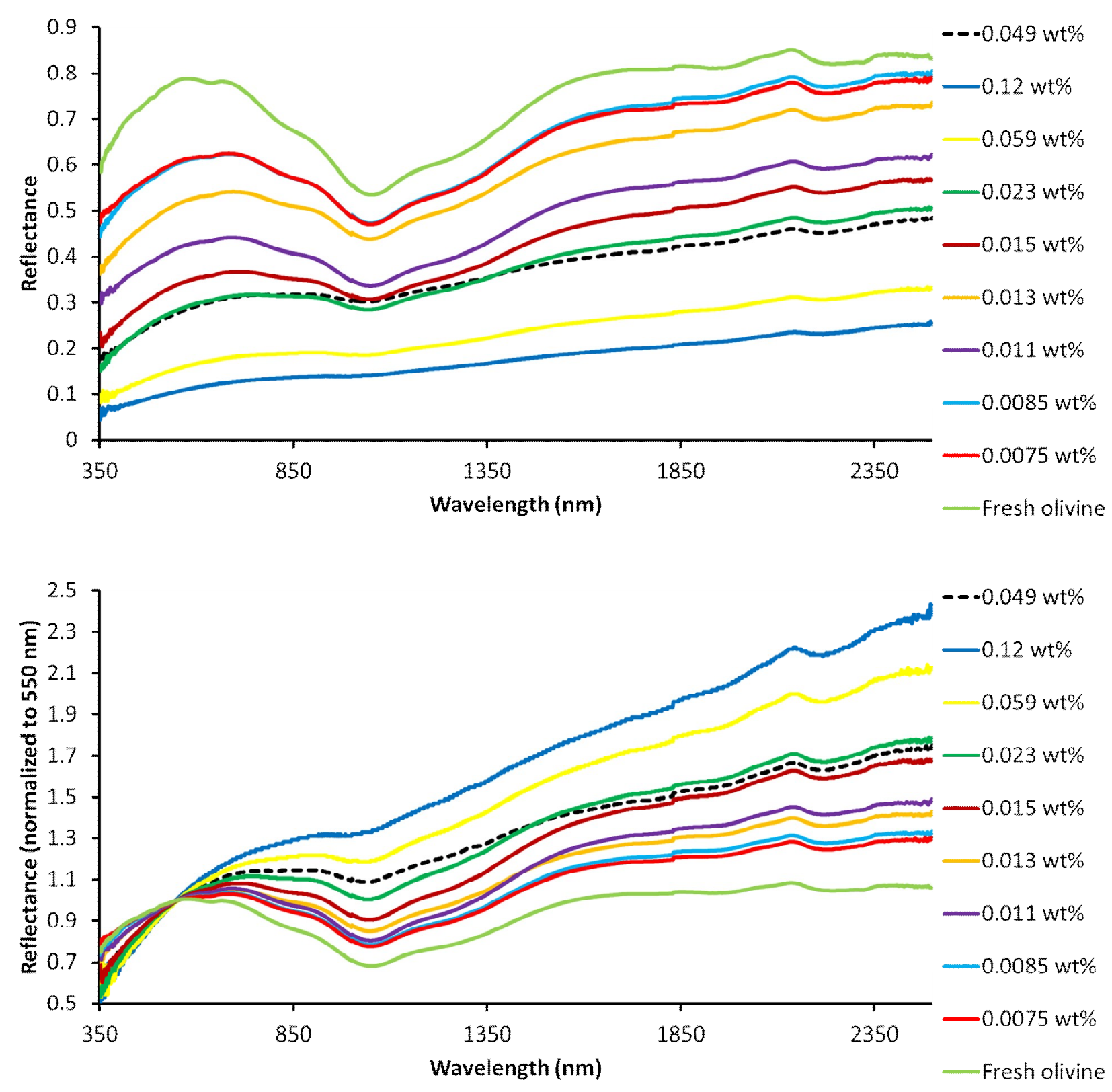
Fig. 7. The linear trend (black line) between the $\mathrm{npFe}^{0}$ amount and the position $1 \mu \mathrm{m}$ band center. $R^{2}$ is the root mean square deviation of the fit.

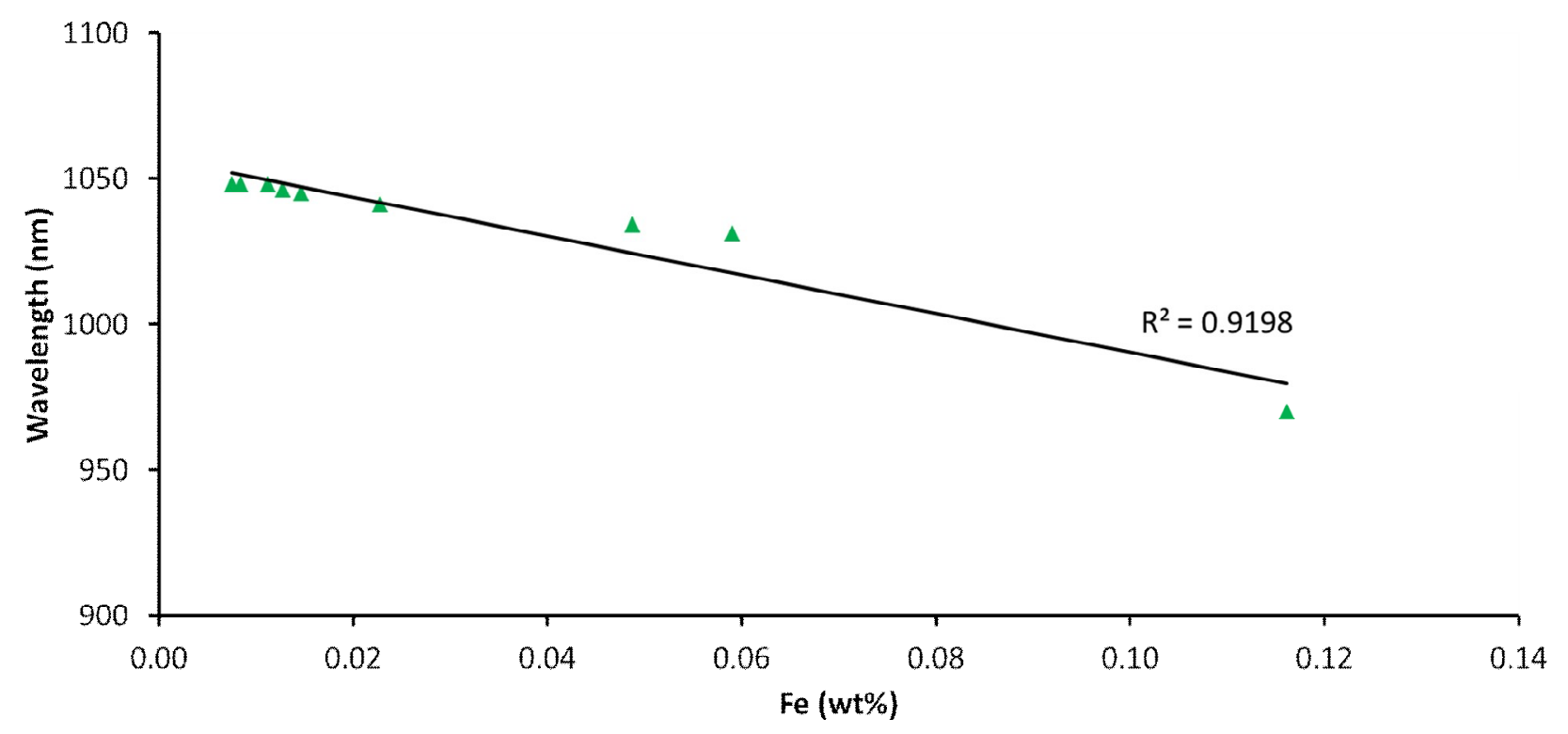


Fig. 8. The logarithmic trend (black lines) between the npFe ${ }^{0}$ amount and the $1 \mu \mathrm{m}$ band depth and the reflectance at $550 \mathrm{~nm} . \mathrm{R}^{2}$ is the root mean square deviation of the fit.

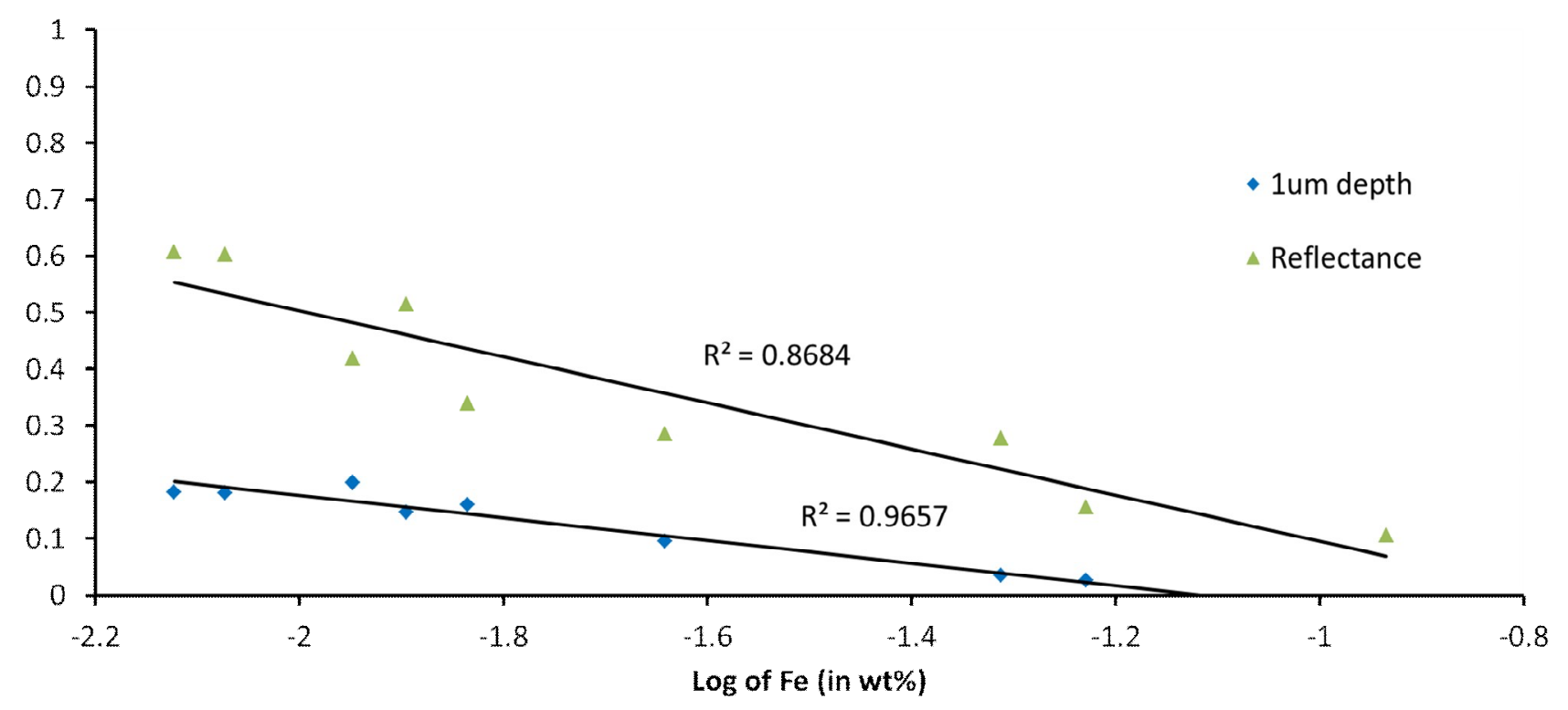


Fig. 9. The logarithmic trend (black line) between the $n p F e^{0}$ amount and the spectral slope. The 850 s30 sample (highlighted by a yellow circle) with additional population of larger npFe ${ }^{0}$ particles does not fully follow the reddening trend. $R^{2}$ is the root mean square deviation of the fit.

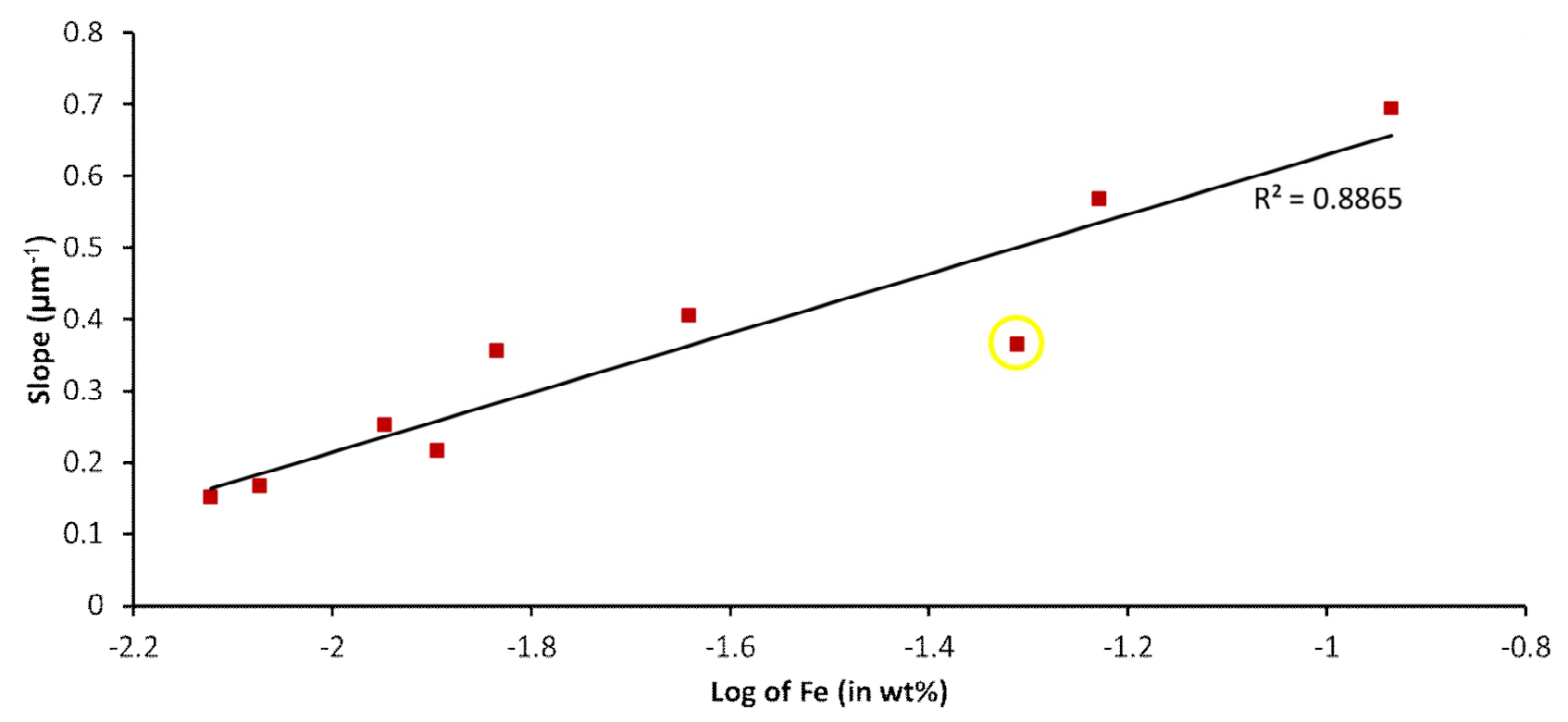


Fig. 10. The logarithmic trend (black line) between the npFe ${ }^{0}$ amount and the $1 \mu \mathrm{m}$ band width at half depth. R2 is the root mean square deviation of the fit.

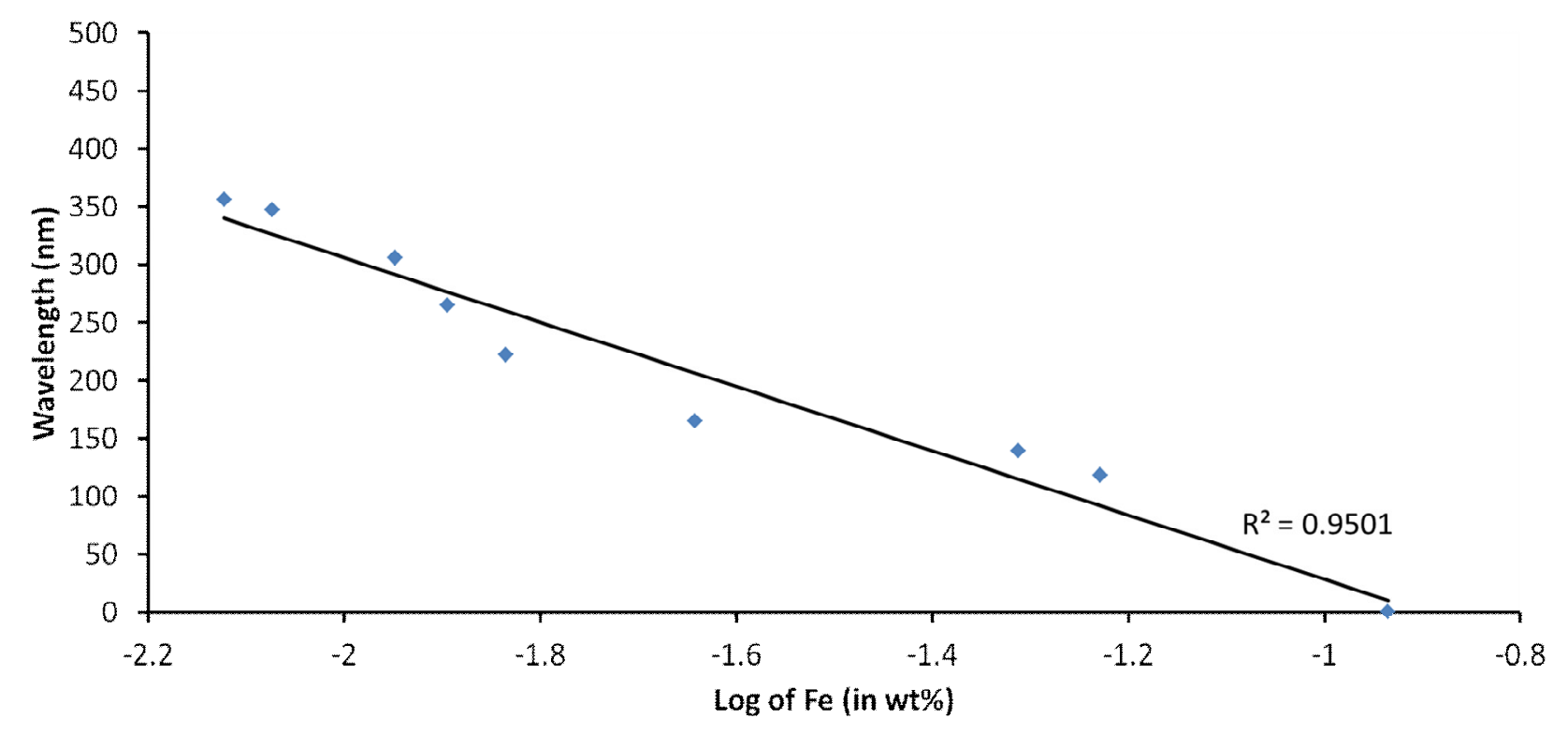


Fig. 11. Positions of the Gaussian band centres (C1-3) as a function of increasing npFe ${ }^{0}$ content.

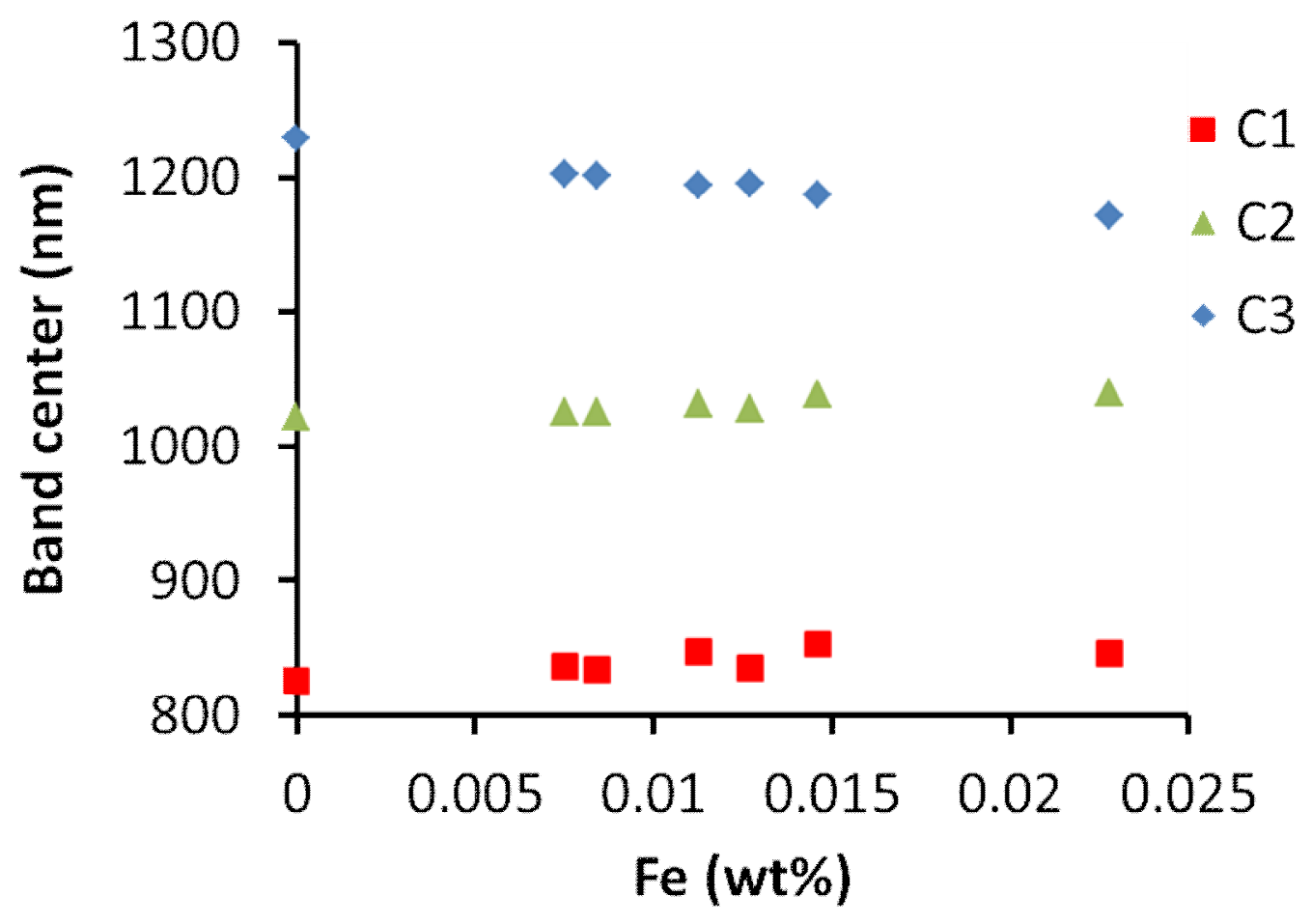


Supplementary material

M odified Gaussian model figures for samples listed in Table 4. Orange - measured spectrum, black

- modeled spectrum, blue - gaussian bands, red - continuum, pink - residual error spectrum.

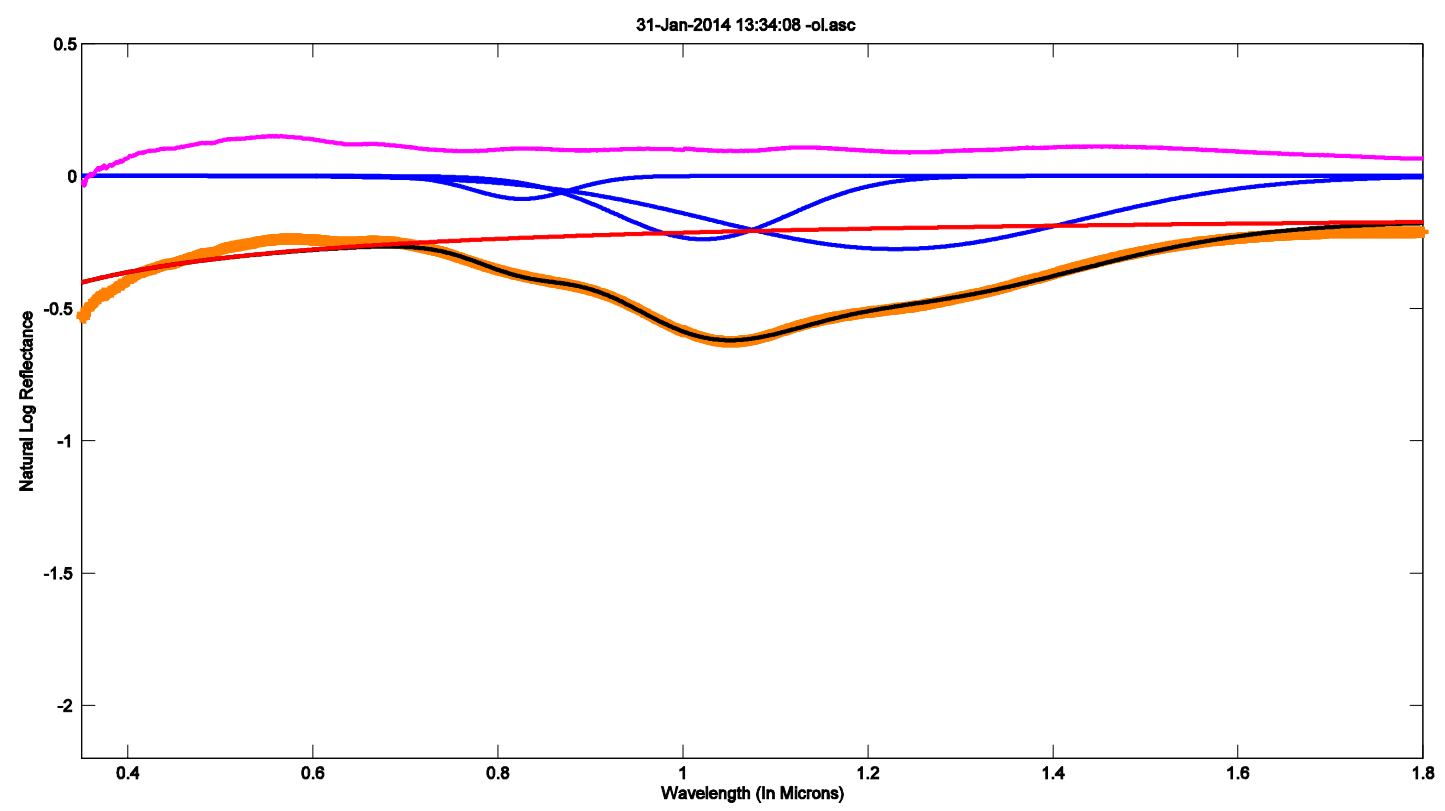

Fig. S1. Fresh olivine. 


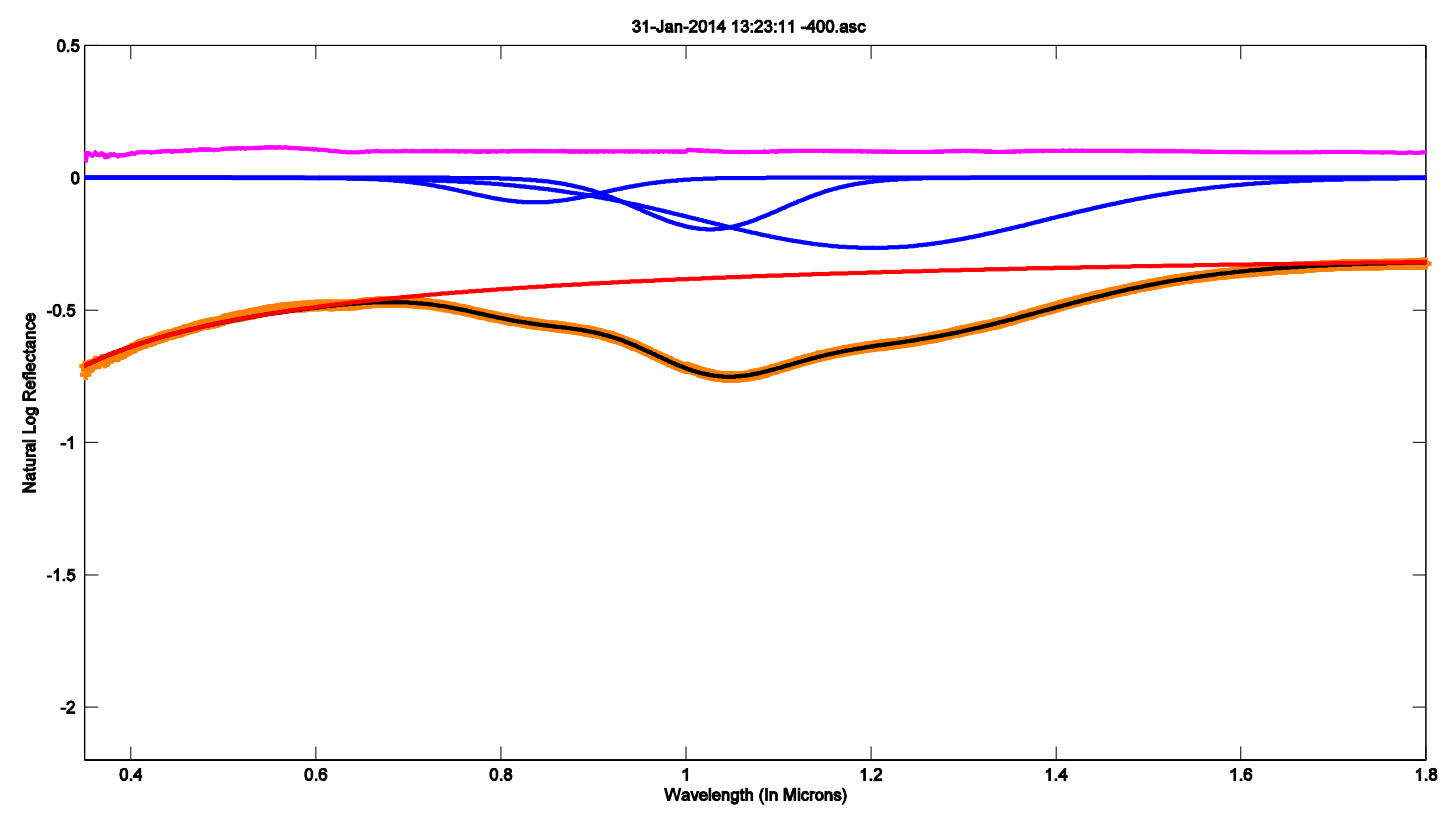

Fig. S2. Sample 400h1.

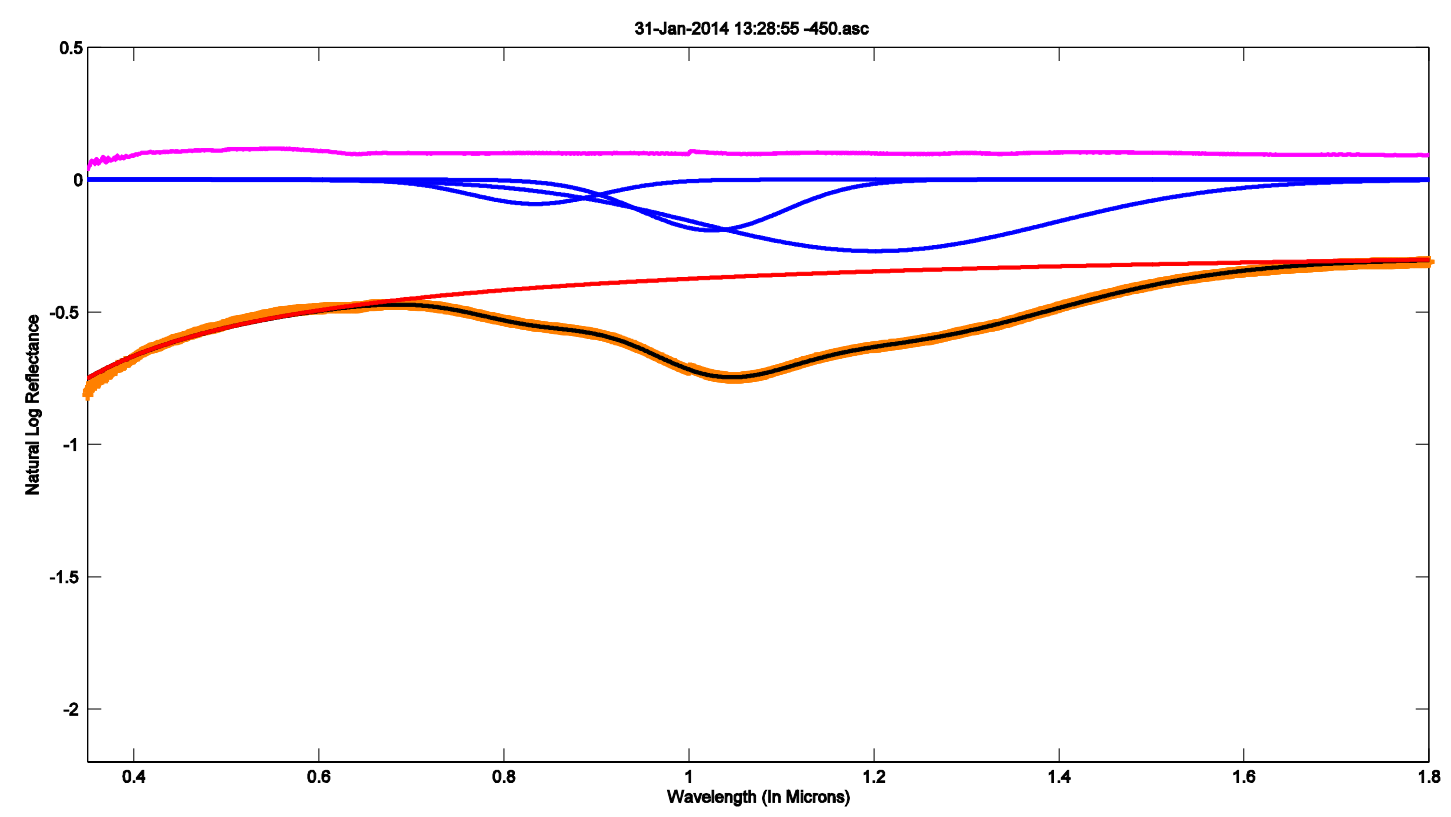

Fig. S3. Sample 450h1. 


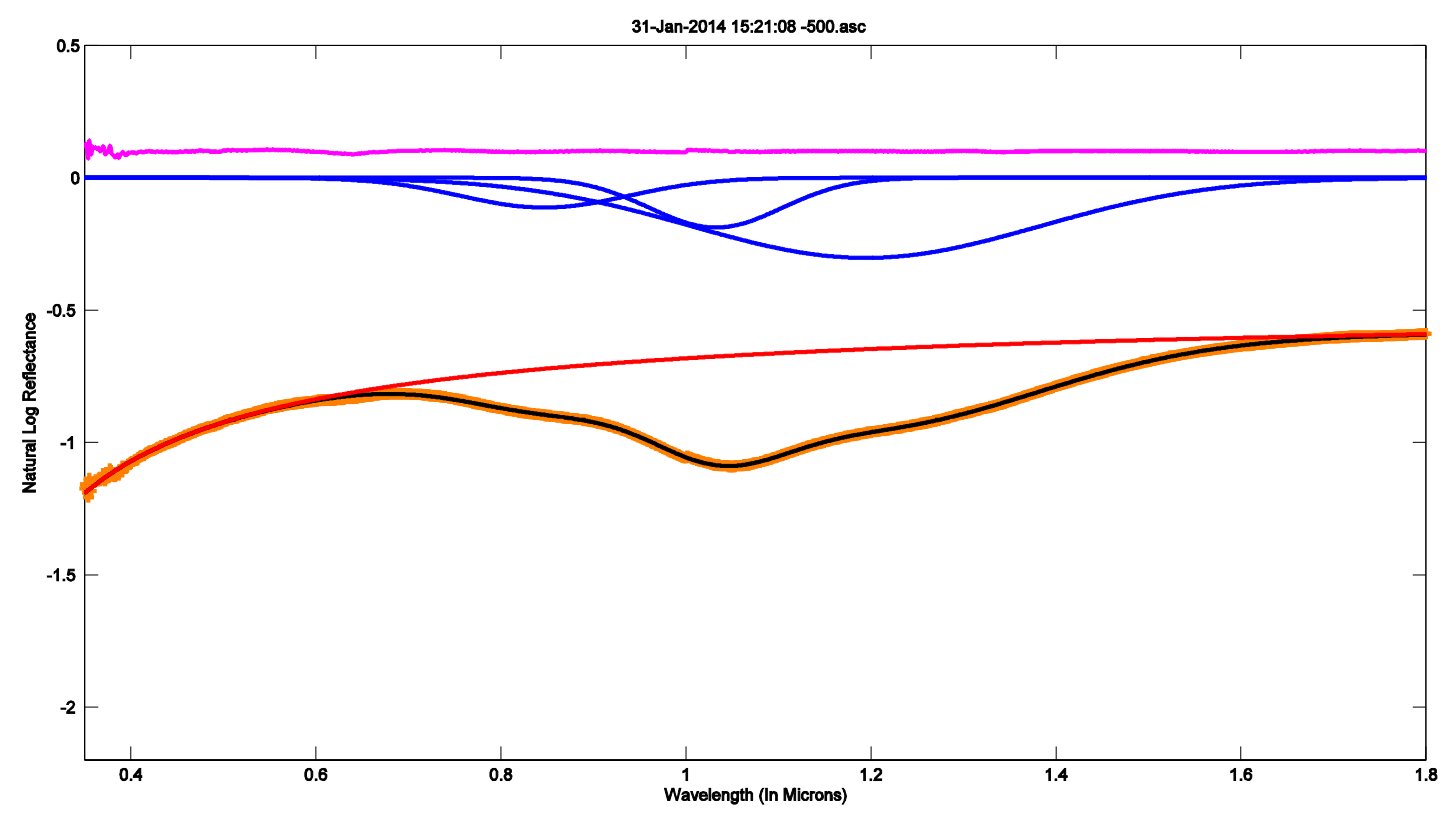

Fig. S4. Sample 500h1.

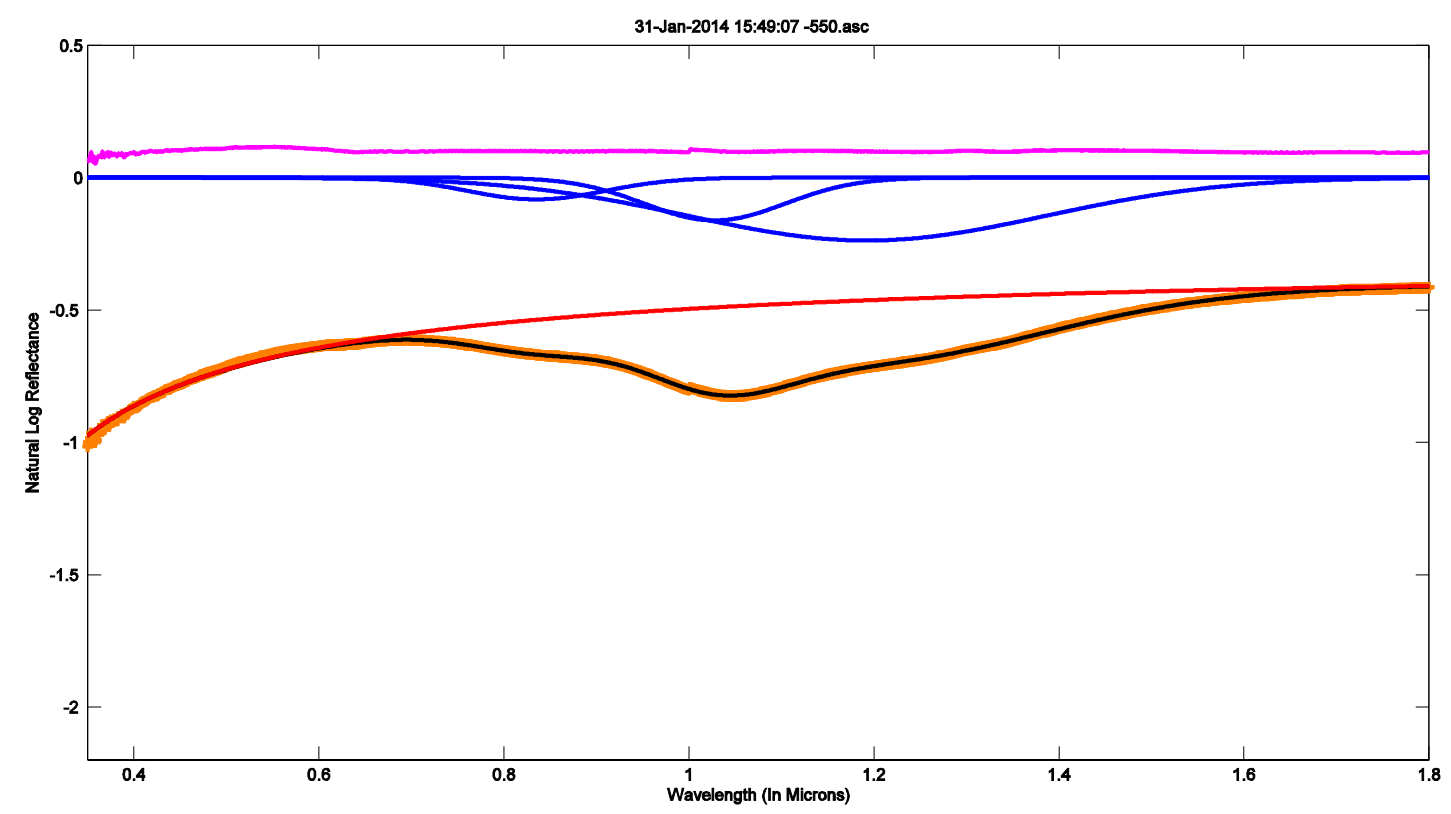

Fig. S5. Sample 550h1. 


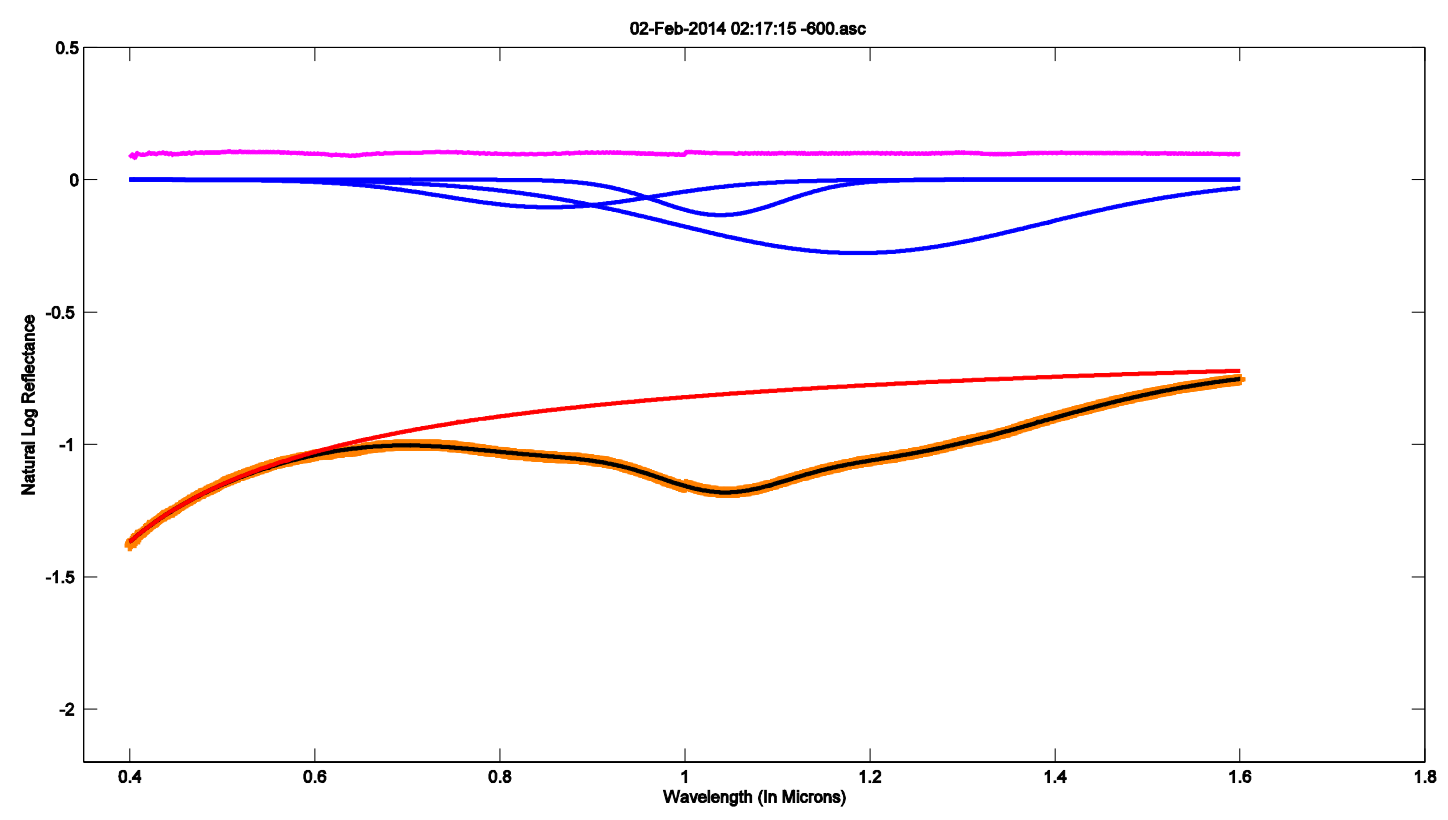

Fig. S6. Sample 600h1.

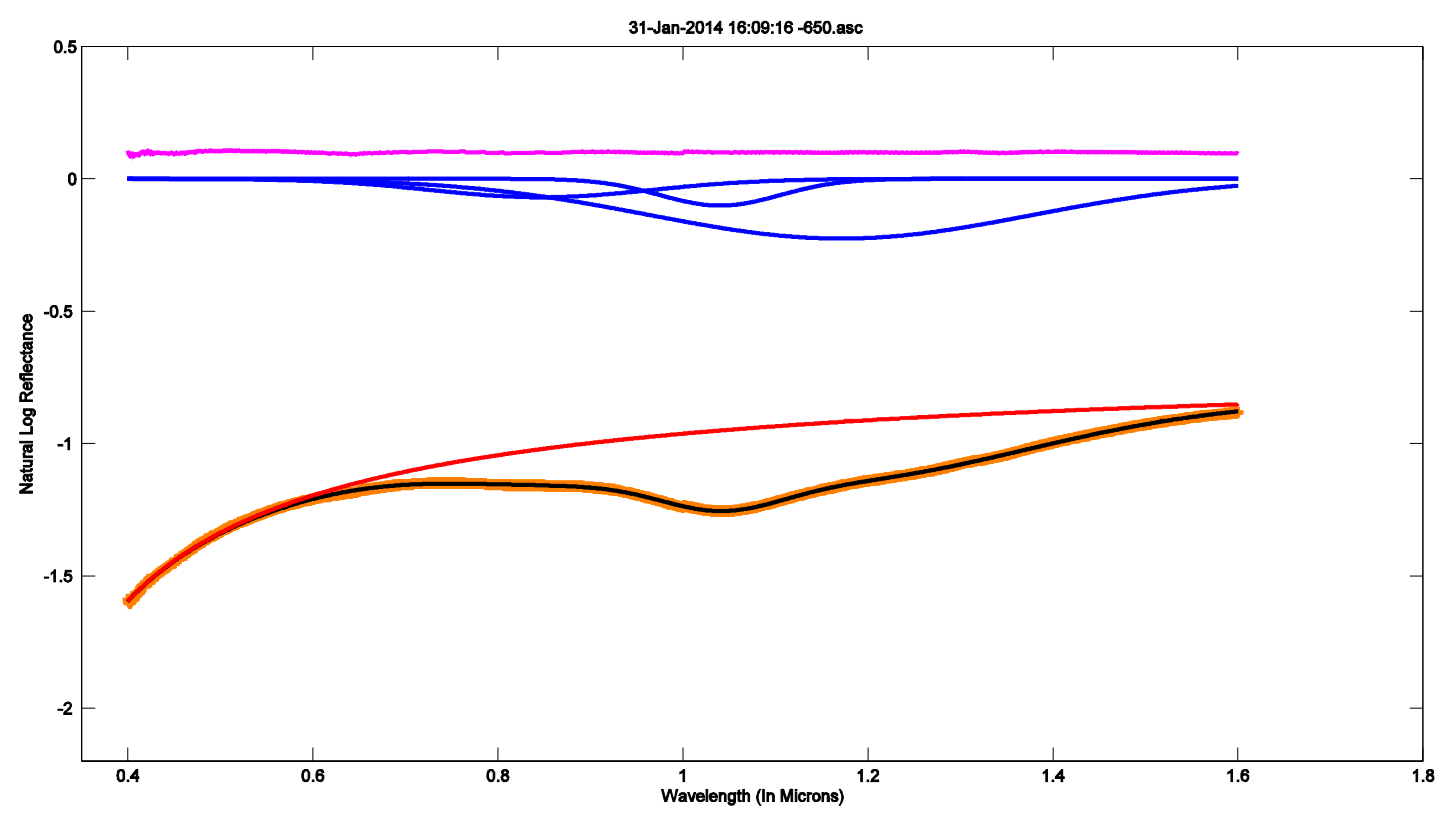

Fig. S7. Sample 650h1. 